\title{
Pro-cachectic factors link experimental and human chronic kidney disease to skeletal muscle wasting programs
}

\author{
Francesca Solagna, ${ }^{1}$ Caterina Tezze, ${ }^{2,3}$ Maja T. Lindenmeyer, ${ }^{1}$ Shun Lu, ${ }^{1}$ Guochao Wu, ${ }^{1}$ Shuya Liu, ${ }^{1}$ Yu Zhao, ${ }^{4}$ Robert Mitchell, ${ }^{5}$ \\ Charlotte Meyer, ${ }^{6}$ Saleh Omairi, ${ }^{7}$ Temel Kilic, ${ }^{6}$ Andrea Paolini, ${ }^{5}$ Olli Ritvos, ${ }^{8}$ Arja Pasternack, ${ }^{8}$ Antonios Matsakas, ${ }^{9}$ \\ Dominik Kylies, ${ }^{1}$ Julian Schulze zur Wiesch, ${ }^{10}$ Jan-Eric Turner, ${ }^{1}$ Nicola Wanner, ${ }^{1}$ Viji Nair, ${ }^{11}$ Felix Eichinger, ${ }^{11}$ Rajasree Menon, ${ }^{11}$ \\ Ina V. Martin, ${ }^{12}$ Barbara M. Klinkhammer, ${ }^{13}$ Elion Hoxha, ${ }^{1}$ Clemens D. Cohen, ${ }^{14}$ Pierre-Louis Tharaux, ${ }^{15}$ Peter Boor, ${ }^{12,13}$
} Tammo Ostendorf, ${ }^{12}$ Matthias Kretzler, ${ }^{11}$ Marco Sandri, ${ }^{2,3}$ Oliver Kretz, ${ }^{1}$ Victor G. Puelles, ${ }^{1,16}$ Ketan Patel,,,17 and Tobias B. Huber,17

'III. Department of Medicine, University Medical Center Hamburg-Eppendorf, Hamburg, Germany. ${ }^{2}$ Veneto Institute of Molecular Medicine, Padua, Italy. ${ }^{3}$ Department of Biomedical Sciences, University of Padova, Padua, Italy. Institute of Medical Systems Biology, University Medical Center Hamburg-Eppendorf, Hamburg, Germany. ${ }^{5}$ School of Biological Sciences, University of Reading, Reading, United Kingdom. ${ }^{6}$ Renal Division, Faculty of Medicine, Medical Centre, University of Freiburg, Freiburg, Germany. ${ }^{7}$ College of Medicine, University of Wasit, Kut, Iraq. ${ }^{8}$ Department of Physiology, Faculty of Medicine, University of Helsinki, Helsinki, Finland. ${ }^{9}$ Molecular Physiology Laboratory, Centre for Atherothrombosis and Metabolic Disease, Hull York Medical School, Hull, United Kingdom. ${ }^{10}$. Department of Medicine, University Medical Center Hamburg-Eppendorf, Hamburg, Germany. " $M$ Michigan Medicine, Ann Arbor, Michigan, USA. ${ }^{2}$ Department of Nephrology and Clinical Immunology and ${ }^{13}$ Institute of Pathology, University Hospital RWTH Aachen, Aachen, Germany. ${ }^{14} \mathrm{Nephrological} \mathrm{Center,} \mathrm{Medical} \mathrm{Clinic} \mathrm{and} \mathrm{Polyclinic} \mathrm{IV,} \mathrm{University} \mathrm{of} \mathrm{Munich,} \mathrm{Munich,} \mathrm{Germany.}{ }^{15}$ Paris Centre de Recherche Cardiovasculaire, INSERM, Université de Paris, Paris, France. ${ }^{16}$ Department of Anatomy and Developmental Biology, Monash University, Melbourne, Australia. ${ }^{77}$ Freiburg Institute for Advanced Studies and Center for Biological System Analysis, University of Freiburg, Freiburg, Germany.

\begin{abstract}
Skeletal muscle wasting is commonly associated with chronic kidney disease (CKD), resulting in increased morbidity and mortality. However, the link between kidney and muscle function remains poorly understood. Here, we took a complementary interorgan approach to investigate skeletal muscle wasting in CKD. We identified increased production and elevated blood levels of soluble pro-cachectic factors, including activin A, directly linking experimental and human CKD to skeletal muscle wasting programs. Single-cell sequencing data identified the expression of activin A in specific kidney cell populations of fibroblasts and cells of the juxtaglomerular apparatus. We propose that persistent and increased kidney production of procachectic factors, combined with a lack of kidney clearance, facilitates a vicious kidney/muscle signaling cycle, leading to exacerbated blood accumulation and, thereby, skeletal muscle wasting. Systemic pharmacological blockade of activin A using soluble activin receptor type IIB ligand trap as well as muscle-specific adeno-associated virus-mediated downregulation of its receptor ACVR2A/B prevented muscle wasting in different mouse models of experimental CKD, suggesting that activin $A$ is a key factor in CKD-induced cachexia. In summary, we uncovered a crosstalk between kidney and muscle and propose modulation of activin signaling as a potential therapeutic strategy for skeletal muscle wasting in CKD.
\end{abstract}

\section{Introduction}

Chronic kidney disease (CKD) affects approximately 10\%-15\% of the world's population (1) and represents a state of progressive and irreversible loss of kidney function (2). Interestingly, two-thirds of adults living with CKD show signs of cachexia (3), a wasting syndrome characterized by progressive depletion of skeletal muscle mass that significantly increases morbidity and mortality and compromises patients' quality of life because of loss of autonomy and fatigue $(4,5)$. Given that interventions for CKD-related cachexia are limited to nutritional support, correc-

Authorship note: VGP, KP, and TBH contributed equally as co-senior authors. Conflict of interest: The authors have declared that no conflict of interest exists. Copyright: $\odot 2021$, Solagna et al. This is an open access article published under the terms of the Creative Commons Attribution 4.0 International License. Submitted: December 20, 2019; Accepted: April 21, 2021; Published: June 1, 2021. Reference information: J Clin Invest. 2021;131(11):e135821. https://doi.org/10.1172/JCl135821. tion of acidosis, physical exercise (6), and experimental pharmacological therapies (e.g., recombinant human growth hormone) (7), a better understanding of the pathophysiological processes that govern this condition may facilitate the discovery of innovative and effective therapies.

The kidney plays a pivotal role in the maintenance of body homeostasis, not only by regulating blood pressure and fluid balance and clearing waste products, but also by acting as a major endocrine organ (8). For example, it is well known that the development of tubulointerstitial fibrosis compromises renal erythropoietin production, resulting in anemia (9). Also, kidney injury may lead to the release of circulating signals that directly affect multiple structures, including central and peripheral vasculature, myocardium, skeleton, and lungs (10-12). Importantly, a direct association between kidney damage and muscle wasting is yet to be established.

Cachexia-induced muscle loss is generally explained by reduced protein synthesis, increased degradation, or a relative imbalance of the two (13). Different hormones and cytokines 
induce muscle wasting through the activation or inhibition of intersecting intracellular signaling pathways (14). In particular, proinflammatory cytokines (e.g., IL-6, IL-1 $\beta$, and TNF- $\alpha$ ) trigger muscle wasting by activating pathways such as NF- $\kappa \mathrm{B}$ and JAK/STAT $(15,16)$. Furthermore, a major cause of muscle loss is driven by an impairment in insulin-like growth factor 1/insulin/PI3K/AKT signaling (17). Activin A is also a key negative regulator of muscle growth that inhibits muscle growth and promotes its degradation $(18,19)$. Together, these signaling networks have been described in association with multiple chronic diseases, including chronic heart failure $(20,21)$, cancer (22), and obstructive pulmonary disease (23). Importantly, the molecular mechanisms involved in CKD-related muscle wasting have not been fully elucidated, and their characterization is essential for the development of novel therapeutic strategies.

This study represents a most comprehensive multidimensional characterization of muscle wasting in the context of CKD. Our findings unravel a potentially new kidney/muscle axis linking $\mathrm{CKD}$ to an increased production of multiple pro-cachectic factors, including activin A, which accumulate in the blood of 3 experimental mouse models and samples from human patients with CKD, resulting in skeletal muscle wasting. This process is exacerbated by a diminished capacity of kidney filtration and, thereby, reduced clearance of circulating activin A. Pharmacological blockade of activin A as well as downregulation of its receptor ACVR2A/B in the muscle were sufficient to overcome all other regulated signals, and to normalize muscle molecular profiles, suggesting that activin $\mathrm{A}$ is a central factor in CKD-induced cachexia and providing a promising therapeutic target. In summary, we have identified and characterized a potentially novel molecular crosstalk between kidney and muscle that may pave the way for pharmacological modulation of skeletal muscle wasting in patients with CKD.

\section{Results}

Kif $3 a^{4 T u b}$ mice develop CKD and cachexia with skeletal muscle atrophy and muscle weakness. We used a genetic mouse model of CKD where the kinesin family member 3A (Kif3a) alleles were deleted in renal tubular epithelial cells (24). Tubular Kif3a deficiency (Kif $3 a^{\Delta \mathrm{Tub}}$ ) led to spontaneous renal cyst formation that almost completely replaced the normal renal parenchyma by 6 weeks of age (Figure 1A). Kidneys from Kif3 $a^{\Delta \text { Tub }}$ mice showed substantial enlargement, accounting for approximately $20 \%$ of total body weight (Figure 1B). Histological staining showed big cysts, dilated tubules, and only a handful of survivingglomeruliin Kif $3 a^{\Delta \text { Tub }}$ kidney compared with wild-type (WT) mice (Supplemental Figure 1A; supplemental material available online with this article; https://doi.org/10.1172/JCI135821DS1), which was reflected in a significant loss of renal function (Figure 1C). These findings support that Kif $3 a^{\Delta \mathrm{Tub}}$ mice showed functional and structural signs consistent with advanced CKD.

Interestingly, despite the dramatic increase in kidney size, Kif $3 a^{\Delta \mathrm{Tub}}$ mice presented significant weight loss (Figure 1D). Systematic analysis of 4 skeletal muscles, including gastrocnemius (GC), tibialis anterior (TA), extensor digitorum longus (EDL), and soleus, showed a significant weight reduction in Kif $3 a^{\Delta T u b}$ compared with WT mice (Figure 1, E and F). Furthermore, we observed a reduced number of fibers in EDL and soleus muscles in Kif3 $a^{\Delta \mathrm{Tub}}$ mice (Supplemental Figure 1, B and C) without features of myofiber degeneration (e.g., centrally nucleated fibers) (Supplemental Figure 1D).

Quantification of cross-sectional area of TA muscle showed a leftward shift at the terminal time point in Kif $3 a^{\Delta \mathrm{Tub}}$ muscle compared with WT (Figure 1G), resulting in a large decrease in the average fiber size $\left(1074 \mu \mathrm{m}^{2}\right.$ in WT compared with $661 \mu \mathrm{m}^{2}$ in Kif $\left.3 a^{\Delta \mathrm{Tub}}\right)$. Myofiber size decreased in every myosin heavy chain (MHC) isoform subtype among all muscles (Supplemental Figure $1, \mathrm{E}$ and $\mathrm{H})$. Specific forces generated by EDL muscles were also reduced in Kif3 $a^{\Delta \mathrm{Tub}}$ compared with WT mice (Figure $1 \mathrm{H}$ ). Together, these data show that skeletal muscle of Kif $3 a^{\Delta \mathrm{Tub}}$ mice with CKD undergoes both functional and structural changes consistent with muscle wasting.

Altered protein synthesis, proteasome, and autophagic flux in $C K D$-related muscle wasting. Muscle growth depends on the fusion of muscle stem cells to form myofibers and on protein synthesis that drives fiber hypertrophy (17). Given our observation of decreased muscle fiber number, we quantified the number of muscle stem cells in EDL muscles and found a $60 \%$ reduction in Kif $3 a^{\Delta \mathrm{Tub}}$ (Figure 2A). In vitro, Kif $3 a^{\Delta \mathrm{Tub}}$ stem cells were able to follow their normal program of differentiation and showed a similar proportion of myogenic positive cells as those from WT mice (Figure $2 \mathrm{~B}$ ). Furthermore, we identified a strong reduction in mRNA levels of insulin-like growth factor 1 (Igf1), a key regulator of cellular proliferation, in muscles from Kif $3 a^{\Delta \mathrm{Tub}}$ compared with WT mice (data not shown).

Skeletal muscle wasting can also be explained by an imbalance between protein synthesis and degradation. The capacity for muscle protein synthesis was assessed in vivo using a puromycin incorporation analysis (Surface Sensing of Translation, SUnSET; ref. 25), which revealed a strong impairment of protein production in muscles from Kif $3 a^{\Delta \mathrm{Tub}}$ mice (Figure $2 \mathrm{C}$ ). Then, we investigated the status of the ubiquitin-proteasome and autophagy-lysosome systems. Atrophy-related ubiquitin ligases Atrogin-1, Musa, Murf1, Itch, and Fbxo31 were all upregulated in muscles from Kif $3 a^{\Delta \mathrm{Tub}}$ mice (Supplemental Figure 2A). Furthermore, the expression of several genes associated with autophagy, including Bnip3, Becn1, and Ambra1, was also significantly upregulated in muscles from Kif3 $a^{\Delta \mathrm{Tub}}$ mice (Supplemental Figure 2B).

Colchicine, by destabilizing the microtubule network, blocks the delivery of autophagosomes to lysosomes, resulting in the accumulation of autophagic vacuoles, and therefore, is an excellent tool for monitoring autophagy flux in vivo (26). Colchicine treatment led to a stronger accumulation of lipidated LC3 and p62 in muscles from Kif $3 a^{\Delta \mathrm{Tub}}$ mice, suggesting autophagy activation (Figure 2D). Together, these findings show a shift in the balance from muscle synthesis to muscle degradation.

Altered mitochondrial structure and function in CKD-related muscle wasting. Next, we examined the metabolic status of muscle fibers by profiling the proportion of fibers displaying high levels of succinate dehydrogenase (SDH) activity. EDL and soleus muscles from $K$ if $3 a^{\Delta \mathrm{Tub}}$ mice contained a lower proportion of oxidative fibers (Figure 2E and Supplemental Figure 2C) and a lower expression of genes encoding respiratory chain complex (RCC) proteins (Supplemental Figure 2D) compared with WT mice. While mitochondria density in both subsarcolemmal (SS) and intramy- 
A

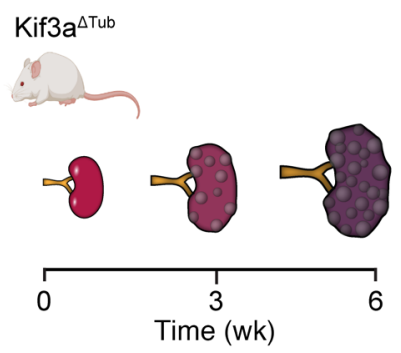

E

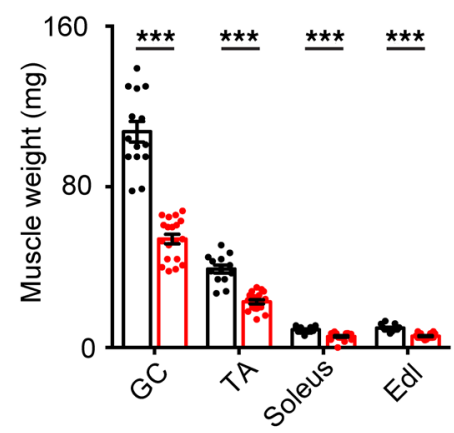

B

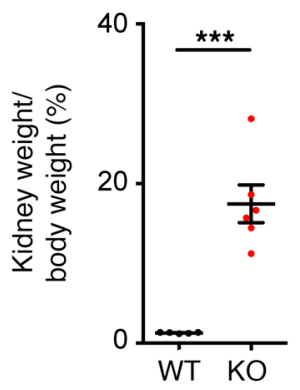

$\mathbf{F}$

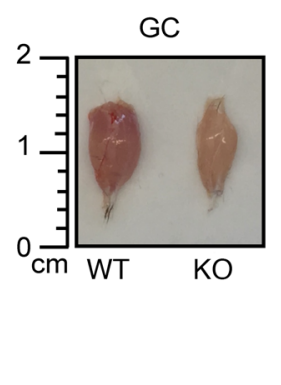

C

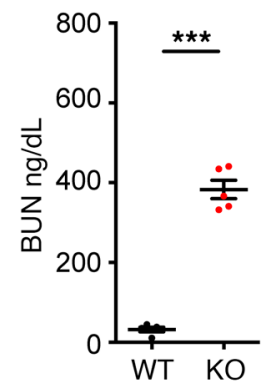

G

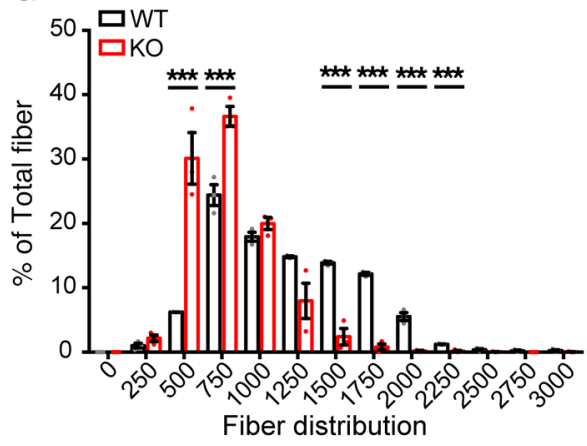

D

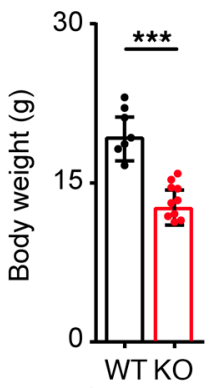

H

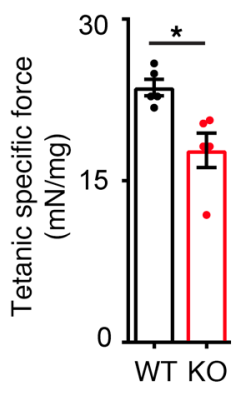

Figure 1. Experimental CKD induces loss of muscle mass and function. (A) Schematic representation of cystic mouse model. (B) Kidney weight/body weight expressed as a percentage and a representative image of the 6-week kidney of WT and Kif3a $a^{\Delta T u b}(n=5$ mice). (C) Blood urea nitrogen (BUN) measurement ( $n=5$ mice). Body weight (D) $\left(n=10\right.$ mice) and muscle tissue weights (E) were measured in WT and Kif3a $a^{\Delta \text { tub }}$ mice $(n=14$ mice). (F) Representative image of the 6-week GC muscle of WT and Kif3a $a^{\Delta T u b}$ mice. (C) Frequency histogram showing the distribution of cross-sectional areas $\left(\mu \mathrm{m}^{2}\right)$ in TA muscle of WT and Kif3a $a^{\Delta \text { tub }}$ mice ( $n=3$ mice). (H) Contractile properties of the EDL muscle $(n=5$ mice). Specific force denotes tetanic force normalized to wet muscle mass. Data shown as mean \pm SEM of 3 independent experiments. Student's $t$ test used for statistical significance. ${ }^{*} P<0.05,{ }^{* * *} P<0.001$.

ofibrillar locations was decreased in muscles from Kif3a $a^{\Delta T u b}$ mice, mitochondria also appeared enlarged (Figure 2F). Furthermore, muscles from Kif $3 a^{\Delta \mathrm{Tub}}$ mice showed a significant increase in the expression of key genes involved in the oxidative stress and mitochondrial unfolded protein response (UPR ${ }^{\mathrm{mt}}$; Figure $2 \mathrm{G}$ ).

Increased kidney production of pro-cachectic factors in CKD-related muscle wasting. In light of a potential crosstalk between kidney and muscle during CKD, the production of renal soluble factors with known pro-cachectic effects was investigated. First, we performed microarray analysis of gene expression in kidneys from WT and Kif $3 a^{\Delta \mathrm{Tub}}$ mice and identified 9 genes with procachectic potential (Figure 3A). From these genes, we decided to focus on inhibin beta-A (Inhba), which encodes activin A, based on its key role as a negative regulator of skeletal muscle mass (18, 19). Next, we checked if the increase of Inhba specifically occurred in the kidney. Interestingly, we found that upregulation of Inhba occurred specifically in the kidney and not in other tissues, such as muscle, liver, and heart (Figure 3B).

To identify the cell population expressing Inhba in the kidney, we performed single-cell RNA sequencing on 5-week-old Kif $3 a^{\Delta \text { Tub }}$ and control kidneys. A total of 6779 cells were profiled after data preprocessing and quality controls and classified into 17 distinct cell types in the Kif3a $a^{\Delta \mathrm{Tub}}$ kidney (Figure $3 \mathrm{C}$ ). We found that Inhba was expressed in a subpopulation of fibroblasts (reference name: Fib-2), which was only found in the Kif $3 a^{\Delta T u b}$ kidney, and in cells from the juxtaglomerular apparatus (reference name: JGA) (Figure 3D). In the control kidney, we found the expression of Inhba only in cells from the JGA (Figure 3, E and F and Supplemental Figure 3A). Furthermore, publicly available single-nucleus RNAsequencing data on fibrotic kidneys from mice 14 days after unilateral ureteral obstruction surgery (27) supported our finding that Inhba was expressed in a subpopulation of fibroblasts (Act. Fib1) and in cells from the JGA (Supplemental Figure 3, B and C). Effective kidney production of activin A would need to be reflective of increased circulating levels. Indeed, we found that blood levels of activin A were significantly elevated in Kif $3 a^{\Delta T u b}$ compared with WT mice (Figure 3G).

Overall, these findings suggest that the kidney source of Inhba is a subpopulation of fibroblasts and cells from the JGA, which are expanded during the development of kidney fibrosis.

Activin $A$ is increased in patients with CKD. To validate our findings from our experimental model of CKD, we analyzed the blood levels of activin A in a first cohort of patients diagnosed with different glomerulonephritis at different stages of CKD $(n=75$; Supplemental Figure 4A). Patients with CKD had significantly higher blood levels of activin A compared with healthy controls $(n=23$; Figure 4A). Blood levels of activin A strongly correlated with estimated glomerular filtration rate (eGFR; $R=$ $-0.56, P<0.0001$; Figure 4B).

A unifying pathological feature of CKD is the development of tubulointerstitial fibrosis. As expected, the percentage of tubulointerstitial fibrosis was inversely associated to eGFR $(R=-0.46 ; P<0.0001$; Figure $4 \mathrm{C})$, while the levels of activin A were directly associated with the percentage of tubulointersti- 
A
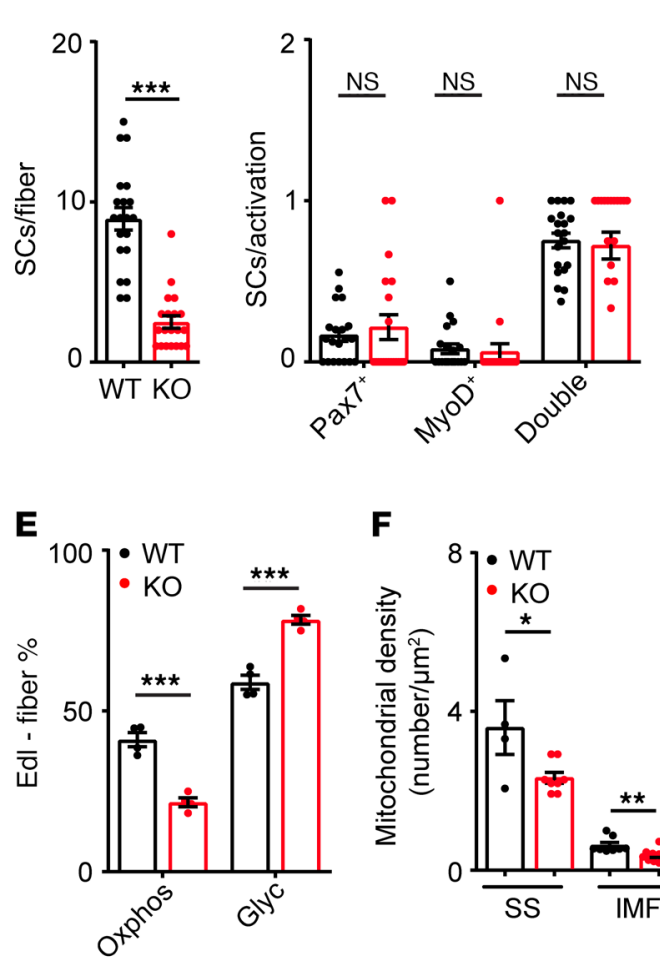

$\mathbf{F}$

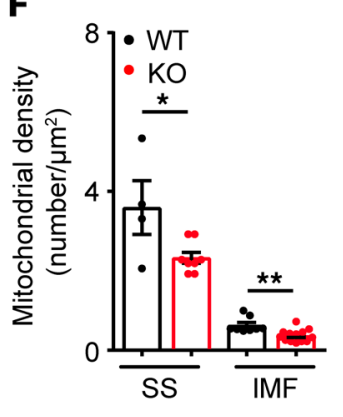

c

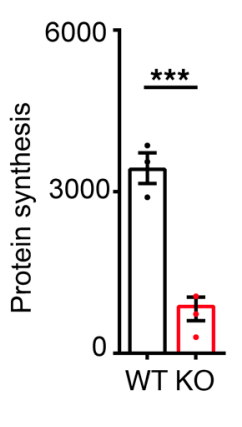

D

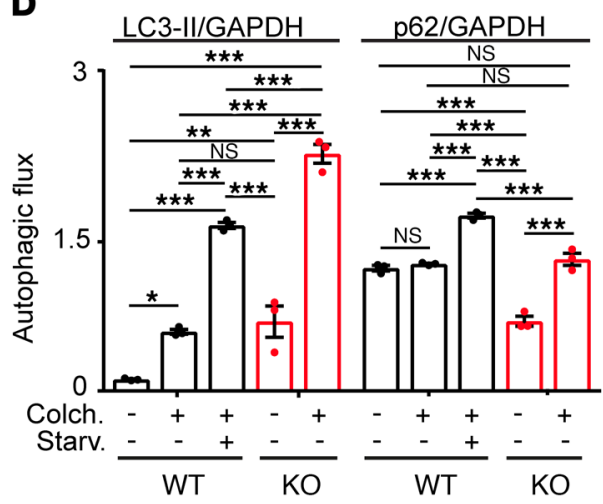

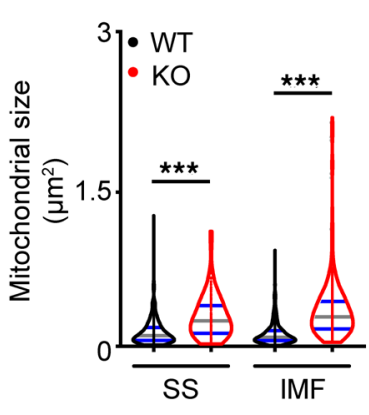

G

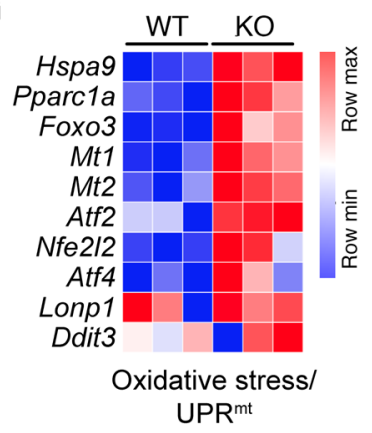

Figure 2. Characterization of CKD-related muscle wasting. (A) Quantification of satellite cell number on freshly isolated EDL fibers ( $n=2$ mice). (B) Profiling of differentiation (WT $n=20, K i f 3 a^{\Delta T u b} n=20$ ). (C) Quantification of puromycin incorporation in GC muscle from 6-week-old WT and Kif3a $a^{\Delta T u b}$ mice,

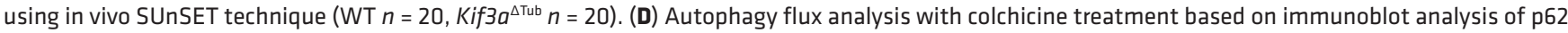
and LC3 of protein extracts from of GC muscles from fed and starved 6-week-old WT and Kif3a $a^{\Delta T u b}$ mice $(n=3$ mice). (E) SDH staining and quantification of EDL muscles of WT and Kif3a $a^{\Delta T u b}$ mice $(n=3$ mice). (F) Quantification of SS and intermyofibrillar (IMF) mitochondrial density and size. One-way ANOVA followed by Bonferroni's multiple-comparison tests (SS: WT $n=4$, Kif3a $a^{\Delta T u b} 8$; IMS: WT $n=8$, Kif3a $a^{\Delta T u b}$ 13). (C) Quantitative gene expression of oxidative stress/UPR ${ }^{m t}$ genes in GC muscle from 6-week-old WT and Kif3a $a^{\Delta \text { Tub }}$ mice $(n=4$ mice). Data shown as mean \pm SEM. Student's $t$ test used for statistical significance, unless otherwise stated. Comparisons of more than 2 groups were calculated using 1-way ANOVA with Tukey's multiple-comparison tests. ${ }^{*} P<0.05 ;{ }^{* *} P<0.01 ;{ }^{* *} P<0.001 ;$ ns, not statistically significant.

tial fibrosis $(R=0.35 ; P<0.01$; Figure $4 \mathrm{D})$. These data suggest that interstitial fibrosis may lead to an increased production of activin $\mathrm{A}$ in human CKD, linked to high circulating levels due to progressive blood accumulation that is potentially amplified by impaired renal function.

Increased production of pro-cachectic factors in patients with $C K D$. In a second cohort of patients with CKD at different stages of CKD $(n=194)$, we analyzed gene expression profiles of human kidney biopsies (Supplemental Figure 4B). Gene expression analysis was performed on microdissected glomerular and tubulointerstitial compartments as previously described (28). mRNA expression of INHBA, nuclear factor $\mathrm{KB} 1$ (NFKB1), CXCL13, and IL-1b was increased in patients with CKD (Figure $4 \mathrm{E}$ and Supplemental Figure $4 \mathrm{C}$ ). Furthermore, mRNA expression of INHBA showed a significant inverse correlation with eGFR $(R=-0.39, P<0.0001$; Figure $4 \mathrm{~F}$ ). Next, we analyzed a third cohort of human kidney biopsies from patients with focal segmental glomerulosclerosis (FSGS; $n=66$; Supplemental Figure 4D). mRNA expression of INHBA, GDF15, IL-1b, NFKB1, CXCL13, and IL-6 showed significant inverse correlations with eGFR (Figure $4 \mathrm{G}$ and Supplemental Figure 4, E and I). Unsupervised cluster analysis of single-cell data in the Kidney Precision Medicine Project reference single-cell RNA-sequencing (scRNA-Seq) data set yielded 31 clusters from
22,268 cells that covered the entire nephron segment along with endothelial and immune cells. Single-cell data showed that INHBA was mainly expressed in vascular smooth muscle/mesangial cells and in this case parietal epithelial cells (Figure 4H). Collectively, these findings suggest a higher renal production of pro-cachectic factor activin $\mathrm{A}$ in patients with CKD.

Activin A-mediated muscle wasting in other models of experimental CKD. Given that patients develop CKD due to multiple underlying causes, it is important to explore other experimental models of CKD, especially those with a strong fibrosis component. First, we used a mouse model of 2,8-DHA-induced nephropathy (adenine nephropathy, AN). By 21 days adenineenriched diet led to progressive kidney disease, characterized by crystal deposits, tubular injury, inflammation, and tubulointerstitial fibrosis (refs. 29, 30; Figure 5, A and B; and Supplemental Figure 5A). Mice with AN had significant weight and lean mass loss over time (Figure 5, C and D).

Systematic analysis of 4 skeletal muscles, including GC, TA, EDL, and soleus, showed a significant reduction of muscle weight in AN compared with WT mice (Figure 5E and Supplemental Figure 5B). Quantification of cross-sectional area of TA muscles showed a leftward shift at the terminal time point in AN compared with WT (Figure 5F), resulting in a large decrease in the average 
A

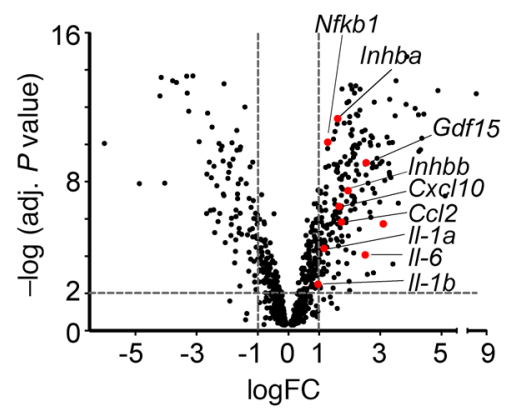

B

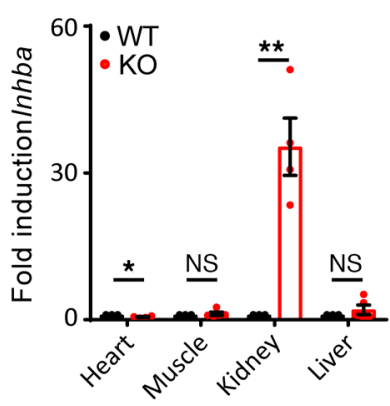

C

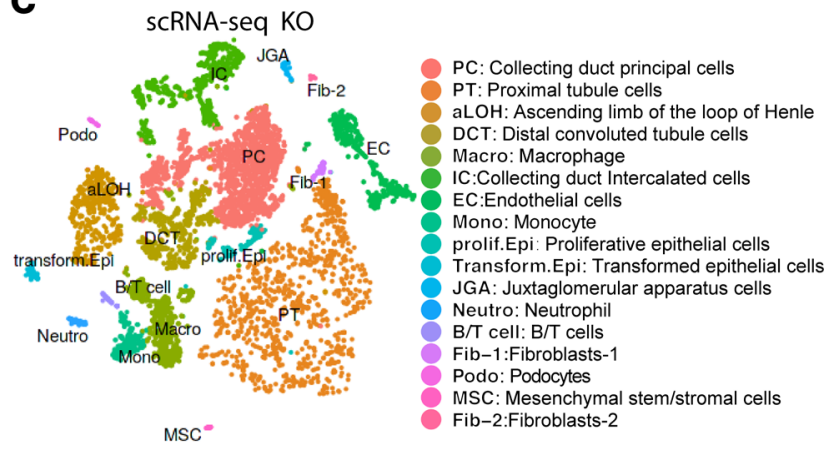

6779 cells
D

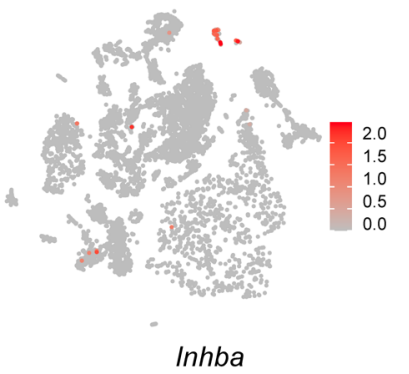

E

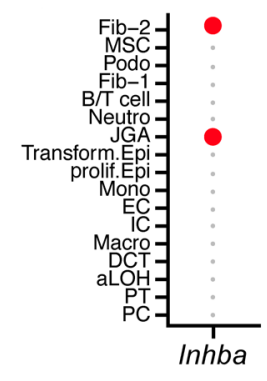

$\mathbf{F}$

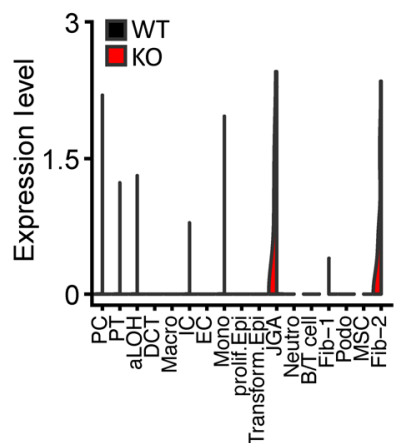

G

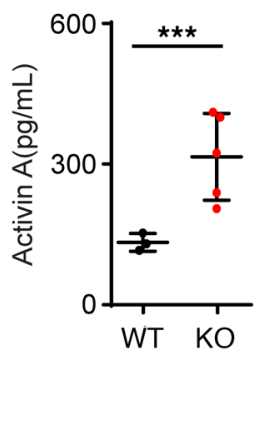

Figure 3. Experimental CKD leads to renal production of soluble pro-cachectic factors. (A) Volcano plot of genes encoding for secreted proteins (red, genes encoding pro-cachectic factors) ( $n=4$ mice). (B) Quantitative reverse transcription PCR (RT-PCR) analysis of $I n h b a$ in different organs ( $n=4$ mice). (C) t-Distributed stochastic neighbor embedding (t-SNE) projection of 6779 cells from the 5 -week Kif3a ${ }^{\Delta T u b}$ kidney demonstrating 17 cell types. (D) t-SNE plot showing the normalized/average expression level of Inhba in Kif3a $a^{\Delta T u b}$ kidney cell types. (E) Dot plot showing the expression of Inhba in the Kif3a ${ }^{\Delta T u b}$ and control kidney. (F) Violin plot showing cluster-specific gene expression of Inhba ( $n=1$ mouse). (C) Blood levels of activin A in WT and Kif3a ${ }^{\Delta T u b}$ mice determined by ELISA (WT $n=3, K O n=5)$. Data shown as mean \pm SEM. Student's $t$ test used for statistical significance, unless otherwise stated. ${ }^{*} P<$ $0.05,{ }^{* *} P<0.01,{ }^{* * *} P<0.001$.

fiber size $\left(1539 \mu \mathrm{m}^{2}\right.$ in WT vs. $1054 \mu \mathrm{m}^{2}$ in AN). Electron microscopy revealed a disorganized sarcomere arrangement with Z-disk misalignment, empty spaces between myofibrils, and dilated endoplasmic reticulum (Figure 5G). Mitochondrial density in both SS and IMF locations was decreased in muscles from AN compared with WT and was associated with mitochondrial enlargement (Figure 5H). Furthermore, gene expression analysis in multiple organs of AN mice revealed an almost exclusive upregulation of mRNA levels of Inhba in the kidney rather than in other tissues, such as muscle, liver, and heart (Figure 5I). Fluorescence in situ hybridization (FISH) supported increased expression of Inhba in the kidney of AN mice compared with WT (Figure 5J). We found a significant increase in gene expression of Acvr2a and $A c v r 2 b$ in the TA muscles of AN mice, suggesting that this pathway is a direct target of activin A (Supplemental Figure 5C). As expected, blood levels of activin A were also significantly elevated in AN compared with WT mice (Figure 5K).

In addition, we also examined a mouse model of Alport syndrome (AS), which is characterized by an altered composition of the glomerular basement membrane that ultimately results in loss of renal function (31). We analyzed tissues from AS mice within the first 8 weeks of life (Supplemental Figure 6A), which already showed significant kidney function impairment (Supplemental Figure 6B) and loss of body weight (Supplemen- tal Figure 6C). Importantly, AS mice showed increased blood levels of activin A compared with WT (Supplemental Figure 6D). At this stage of CKD, there were classical histopathological changes and significant deposition of collagen III and IV (Supplemental Figure 6, $\mathrm{G}$ and $\mathrm{H}$ ).

Collectively, these findings confirm that the increase of blood levels of activin $\mathrm{A}$ is a general response to tubulointerstitial fibrosis during the development of CKD.

Pharmacological inhibition of activin A prevents muscle wasting in experimental CKD. Since increased blood levels of activin $\mathrm{A}$ are a hallmark of CKD in both mice and humans, we decided to test a pharmacological approach to modulate activin A signaling in vivo using a soluble activin receptor type IIB ligand trap (sActRIIB).

Kif $3 a^{\Delta \mathrm{Tub}}$ and WT mice received sActRIIB twice a week by intraperitoneal injections $(10 \mathrm{mg} / \mathrm{kg})$ starting at 2 weeks of age for 4 consecutive weeks (Figure 6A). Treatment with sActRIIB prevented body weight loss in Kif3a $a^{\triangle \mathrm{Tub}}$ mice (Figure $\left.6 \mathrm{~B}\right)$. The weights of all analyzed muscles in sActRIIB-treated Kif3a $a^{\Delta \mathrm{Tub}}$ mice were similar to or even greater than those of WT mice (Figure 6C and Supplemental Figure 7, A and B). Interestingly, the total fiber number loss in EDL muscles was not prevented by sActRIIB (Figure 6D). Quantification of cross-sectional area of TA muscle showed that in mice treated with sActRIIB, fiber sizes were broadly distributed compared with Kif $3 a^{\Delta \mathrm{Tub}}$ mice (Figure $6 \mathrm{E}$ ), resulting in a large 
A

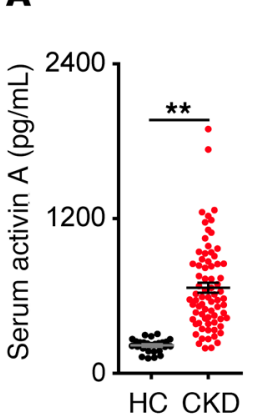

E

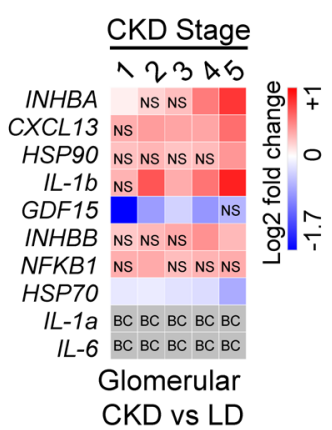

B

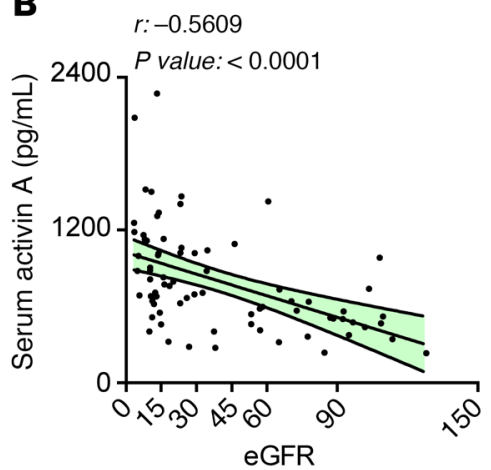

$\mathbf{F}$

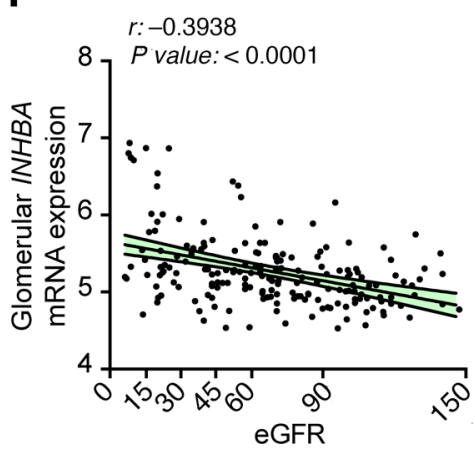

C

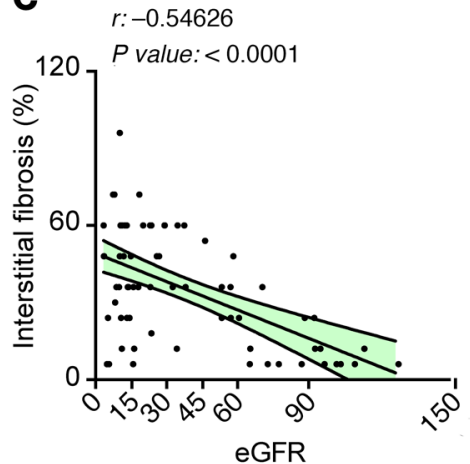

G

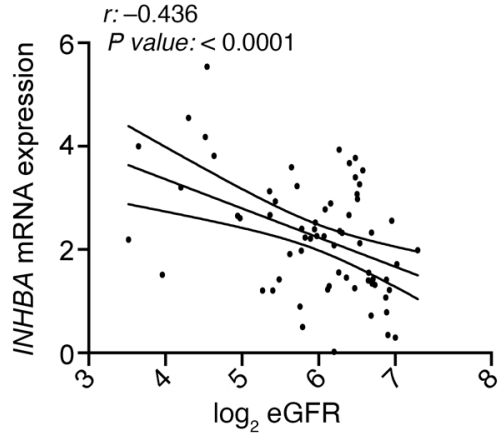

D $\quad r: 0.345$
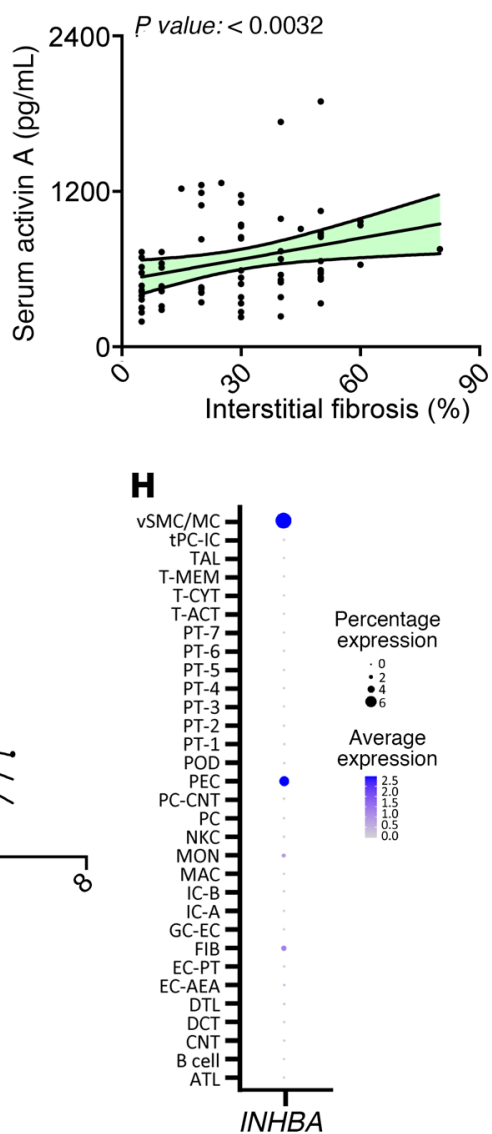

Figure 4. Increased production of pro-cachectic factors in patients with CKD. (A) Activin A blood level in healthy controls and in patients with CKD ( $n=$ 75 patients). (B) Spearman's correlation analysis of serum activin A and eGFR. ${ }^{* *} P<0.01$. (C) Spearman's correlation analysis of IF/TA\% (renal interstitial fibrosis and tubular atrophy) and eGFR. (D) Spearman's correlation analysis of serum activin A and IF/TA\% (renal interstitial fibrosis and tubular atrophy). (E) Log fold change of quantitative gene expression of genes encoding pro-cachectic factors in glomeruli of manually microdissected biopsies from patients with different CKD stages (Clomerular: CKD1: $n=55$; CKD2: $n=52$; CKD3: $n=44$; CKD4: $n=26$; CKD5: $n=10$. Tubular: CKD1: $n=56$; CKD2: $n=46$; CKD3: $n=37$; CKD4: $n=26$; CKD5: $n=10$. Live donor: $n=42$ ). A $q$ value below $5 \%$ was considered statistically significant. Nonsignificantly changed genes are denoted as ns and genes below background cutoff as BC. GDF15, growth differentiation factor 15. (F) Spearman's correlation analysis for glomerular activin A mRNA expression with eGFR. (G) Spearman's correlation analysis of patients with focal segmental glomerulosclerosis (FSGS) of INHBA mRNA expression with $\log _{2}$ eGFR ( $n=66$ patients). (H) Dot plot shows the relative average mRNA expression of INHBA across the 31 clusters identified from the combined analysis of the 24 adult human kidney scRNA-Seq data sets.

increase in the average fiber size $\left(1074 \mu \mathrm{m}^{2}\right.$ in WT vs. $661 \mu \mathrm{m}^{2}$ in Kif $3 a^{\Delta \mathrm{Tub}}$ vs. $1402 \mu \mathrm{m}^{2}$ in sActRIIB-treated Kif $\left.3 a^{\Delta \mathrm{Tub}}\right)$. Furthermore, the increase in fiber cross-sectional area in sActRIIB-treated Kif $3 a^{\Delta T u b}$ mice was confirmed in all fiber types irrespective of their MHC expression (Supplemental Figure 7, C and D). Treatment with sActRIIB normalized the number of muscle stem cells (Figure 6F) but did not impact their differentiation potential (Supplemental Figure 7E). Importantly, treatment with sActRIIB increased EDL tetanic and specific force in Kif $3 a^{\Delta \mathrm{Tub}}$ mice (Figure $6 \mathrm{G}$ and Supplemental Figure 7F).

Next, we evaluated the role of sActRIIB treatment on muscular metabolic status. Treatment with sActRIIB did not significantly alter the portion of oxidative fibers in EDL and soleus of Kif $3 a^{\Delta T u b}$ compared with WT mice (Supplemental Figure 7, G and H). Mitochondrial density in both SS and IMF locations showed a slight increase in Kif $3 a^{\Delta \text { Tub }}$ mice after sActRIIB treatment (Figure $6 \mathrm{H}$ ). This observation was consistent with an increased expression of genes encoding RCC subunits and fatty acid oxidation, which generates direct substrates for electron transport and ATP production (Supplemental Figure 7, I and J). The number of enlarged mitochondria in Kif $3 a^{\Delta T u b}$ mice was significantly reduced after sActRIIB treatment (Figure 6H), which correlated with a decrease in expression of key genes involved in the UPR ${ }^{\mathrm{mt}}$ (Figure 6I).

In Kif $3 a^{\Delta \mathrm{Tub}}$ mice, sActRIIB administration decreased levels of free activin in circulation (Figure 7A), and other pro-cachectic factors (Figure 7B), for example IL-6 and glucocorticoids. Treatment with sActRIIB restored AKT/mTOR signaling (Figure 7, C and $\mathrm{D}$ ) and led to an increase in the protein synthesis that was previously impaired in Kif $3 a^{\Delta \mathrm{Tub}}$ mice (Figure 7E). Treatment with sActRIIB also prevented activation of the activin A/p38 MAPK signaling pathway (Figure $7 F$ ), which contributes to protein degradation in skeletal muscle (32). Next, we examined the regulation of forkhead box protein $\mathrm{O}$ (FoxO), a downstream target of glucocorticosteroids (Supplemental Figure 8A), which is consid- 
A

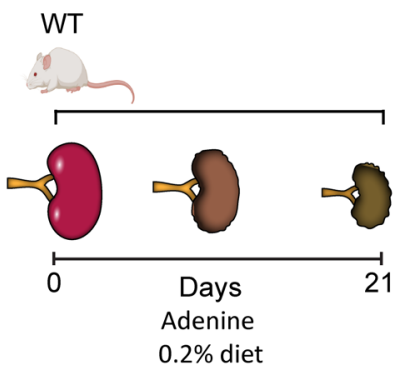

D

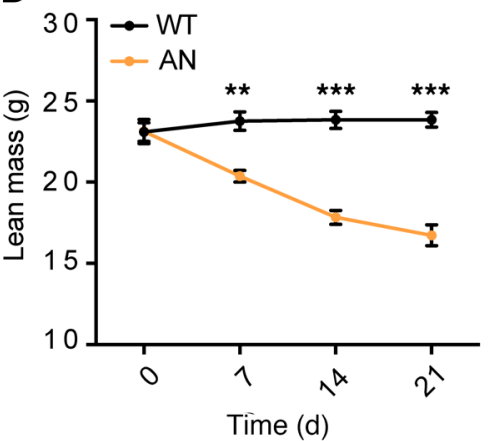

G

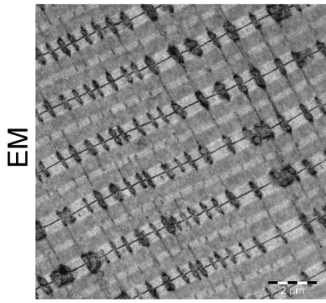

WT

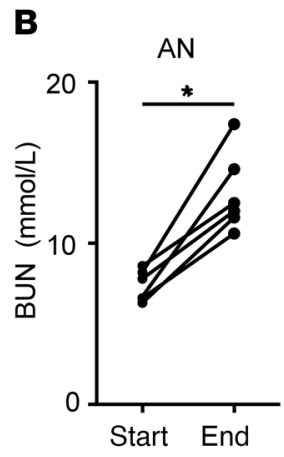

E

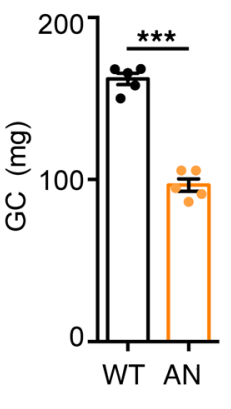

C

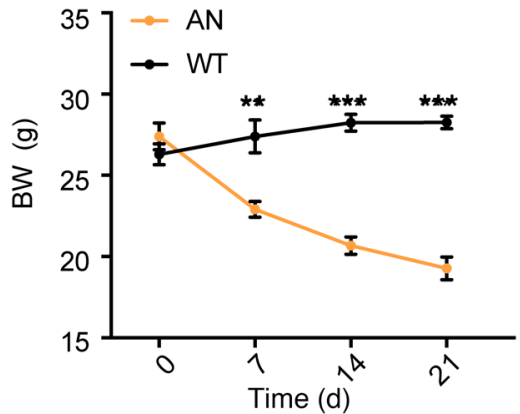

$\mathbf{F}$

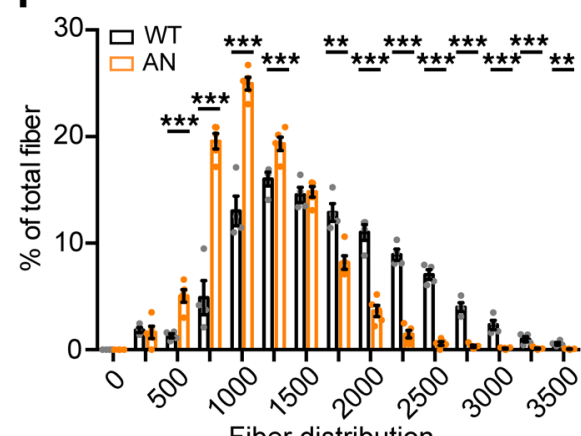

Fiber distribution

H
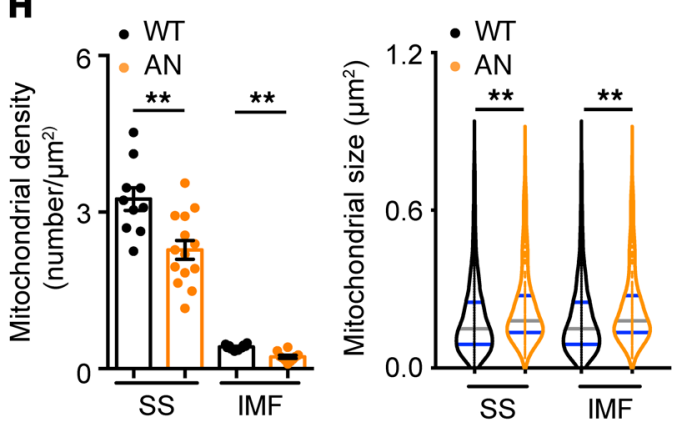

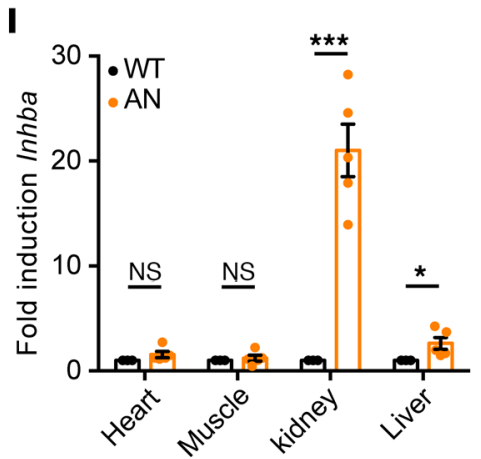

J
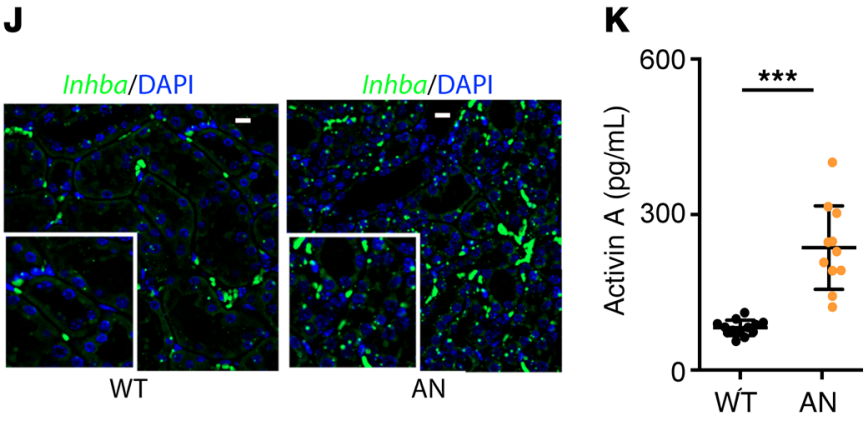

Figure 5. Skeletal muscle atrophy and accumulation of activin A in the blood are common features of different models of kidney fibrosis. (A) Schematic representation of 2,8-DHA nephropathy model (AN): mice are fed for 21 days with adenine-enriched diet. (B) BUN measurement ( $n=5$ mice). (C) Body weight (D) and lean mass curve in WT and AN mice ( $n=5$ mice). (E) GC muscle weights ( $n=5$ mice). (F) Frequency histogram showing the distribution of crosssectional areas $\left(\mu \mathrm{m}^{2}\right)$ in TA of WT and AN mice $(n=4$ mice). (C) Electron micrographs of EDL muscles of WT and AN mice. Scale bar: $2 \mu \mathrm{m}$. (H) Quantification of SS and IMF mitochondrial density and size. One-way ANOVA followed by Bonferroni's multiple-comparison tests. (I) Quantitative RT-PCR analysis of Inhba in different organs ( $n=5$ mice). (J) In situ hybridization was performed with an RNAscope probe targeting Inhba mRNA. Representative images of WT and AN kidney. Inhba mRNA shown in green and nuclei counterstained with DAPI (blue). Scale bars: $10 \mu \mathrm{m}$. (K) Blood levels of activin A in WT and AN mice determined by ELISA ( $n=5$ mice). Values are mean \pm SEM. Student's $t$ test used for statistical significance, unless otherwise stated. ${ }^{*} P<0.05,{ }^{* *} P<0.01,{ }^{* * *} P<0.001$ 
A

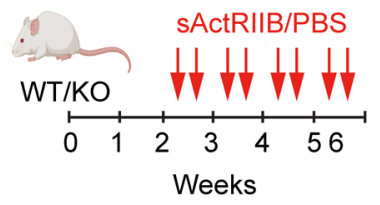

B

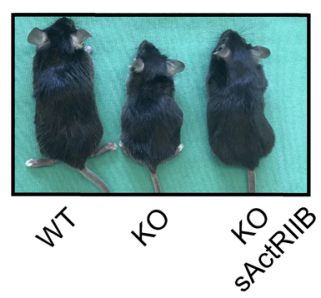

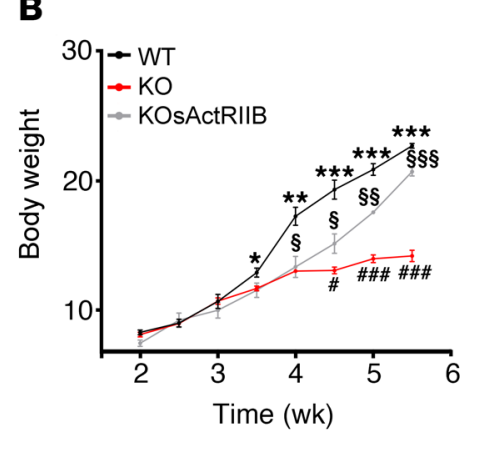

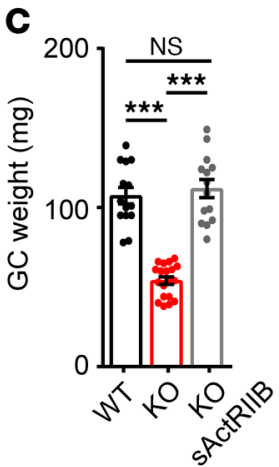

E

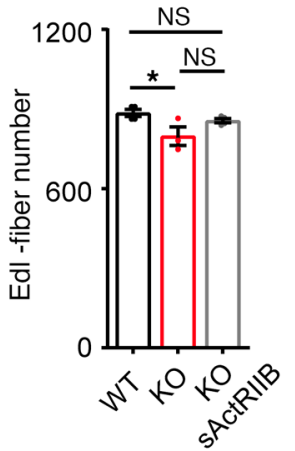

H

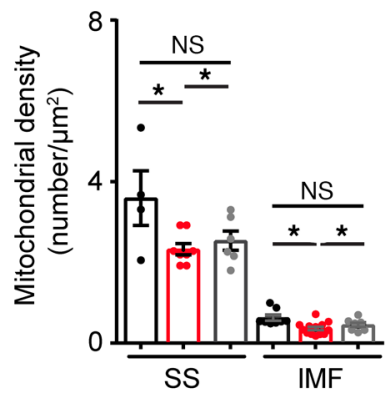

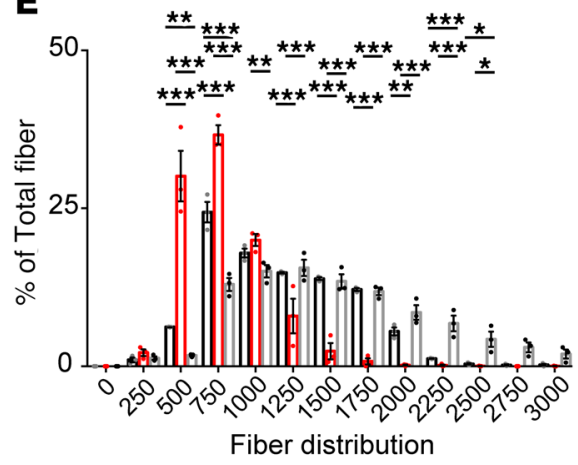

$\mathbf{F}$

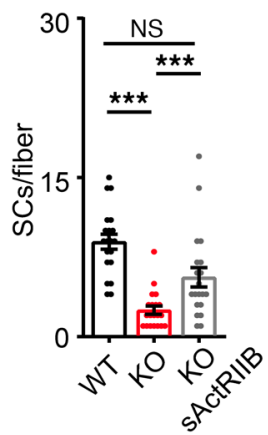

G

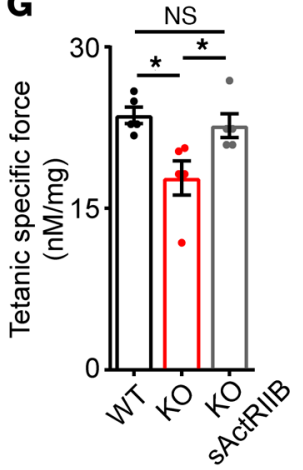

I
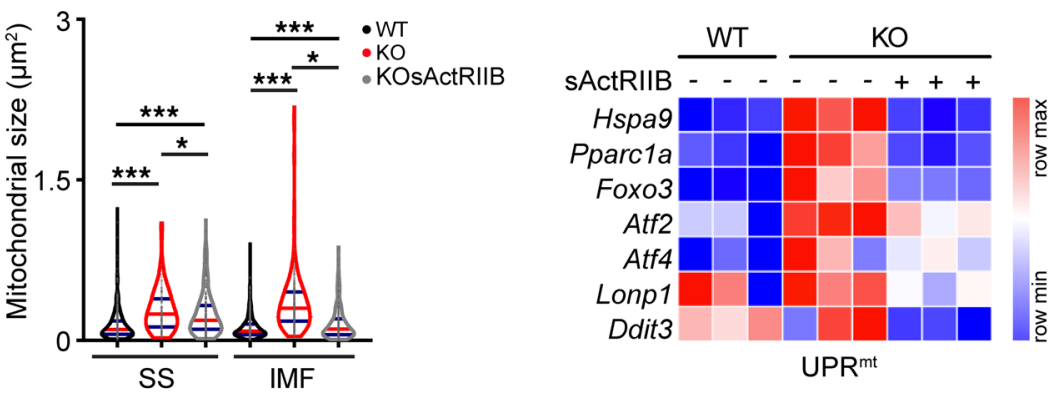

Figure 6. Pharmacological inhibition of activin A prevents muscle wasting in experimental CKD. (A) Schematic representation of experimental design: treatment of WT and Kif3a $a^{\Delta T u b}$ mice twice weekly by intraperitoneal (i.p.) injections of vehicle treatment or $10 \mathrm{mg} / \mathrm{kg}$ sActRIIB starting at $2 \mathrm{weeks}$ of age

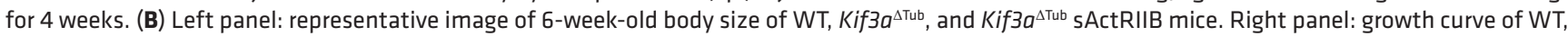
Kif $3 a^{\Delta T u b}$, and Kif3 $3 a^{\Delta \text { Tub }}$ sActRIIB mice ( $n=5$ mice). (C) GC weight of WT, Kif3 $a^{\Delta T u b}$, and Kif3a $a^{\Delta T u b}$ sActRIIB mice $(n=13$ mice). (D) Analysis of the number of fibers per muscle in WT and Kif3a $a^{\Delta T u b}$ mice and Kif3 $a^{\Delta T u b}$ sActRIIB mice $(n=3$ mice). (E) Frequency histogram showing the distribution of cross-sectional areas $\left(\mu \mathrm{m}^{2}\right)$ in TA of WT, Kif3 $a^{\Delta \mathrm{Tub}}$, and Kif3a $a^{\Delta \mathrm{Tub}}$ sActRIIB mice $(n=3$ mice). (F) Quantification of satellite cell number on freshly isolated EDL fibers in WT and Kif3a $a^{\Delta \text { Tub }}$ mice and Kif3a $a^{\Delta T u b}$ sActRIIB mice ( $n=2$ mice). Data of WT and Kif3a $a^{\Delta T u b}$ have already been presented in Figure 2B. (C) Tetanic specific force measurement in EDL muscle of WT and Kif3a $a^{\Delta \top u b}$ mice and Kif $3 a^{\Delta T u b}$ sActRIIB mice. Data of WT and Kif $3 a^{\Delta \top u b}$ have already been presented in Figure $1 \mathrm{H}$ ( $n$ = 5 mice). (H) Quantification of SS and IMF mitochondrial density and size. One-way ANOVA followed by Bonferroni's multiple-comparison tests. Data of WT and Kif $3 a^{\Delta T u b}$ have already been presented in Figure $2 \mathrm{~F}\left(n=3\right.$ mice). (I) Quantitative gene expression of UPR ${ }^{\mathrm{mt}}$ genes in GC muscle from 6-week-old WT, $K i f 3 a^{\Delta T u b}$, and Kif $3 a^{\Delta T u b}$ sActRIIB mice $(n=4$ mice). Data shown as mean \pm SEM of 3 independent experiments. Comparisons of more than 2 groups were calculated using 1-way ANOVA with Tukey's multiple-comparison tests: ${ }^{*}$ when comparing WT vs. Kif $3 a^{\Delta T u b, ~}{ }^{\S}$ when comparing WT vs. Kif3a $a^{\Delta T u b}$ sActRIIB,

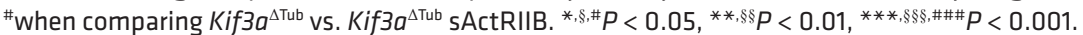

ered a central regulator of muscle homeostasis $(26,33,34)$. We found an increase in both gene expression and protein level of FOXOs that were diminished by sActRIIB treatment (Figure 7G), resulting in a downregulation of FoxO-dependent gene transcription (Supplemental Figure 8B) and the restoration of normal levels of autophagy (Figure 7H). It is also worth noting that ActRIIB signals through a subset of TGF- $\beta$ family ligands, including myostatin $(35,36)$. Importantly, mRNA levels of myostatin in multiple organs (Supplemental Figure 8C) as well as GDF8 blood levels were not changed in Kif $3 a^{\Delta \mathrm{Tub}}$ compared with WT mice (Supplemental Figure $8 \mathrm{D}$ ), suggesting a specific role of activin A in promoting muscle wasting in CKD-induced cachexia that can be pharmacologically targeted.

In vivo blockade of activin A attenuates experimental CKD. Administration of sActRIIB significantly reduced kidney/body weight ratios in Kif3a $a^{\Delta \mathrm{Tub}}$ mice (Supplemental Figure 9A), showing a partial protec- 
A

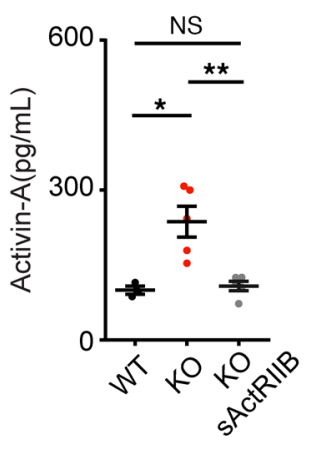

B

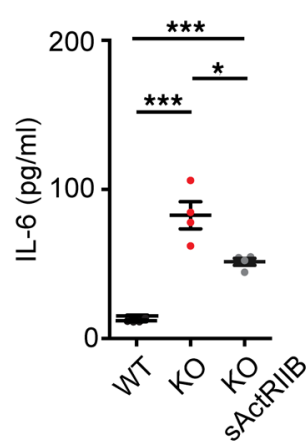

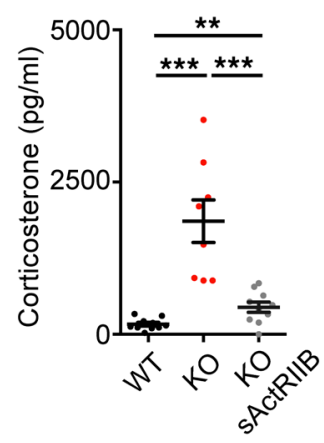

C

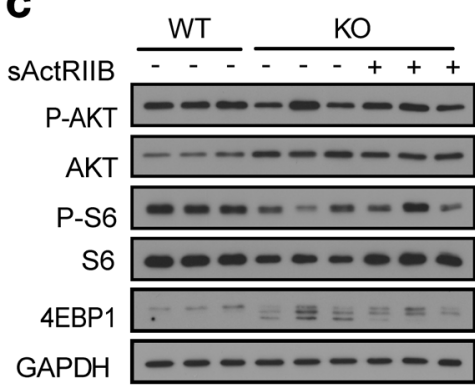

D

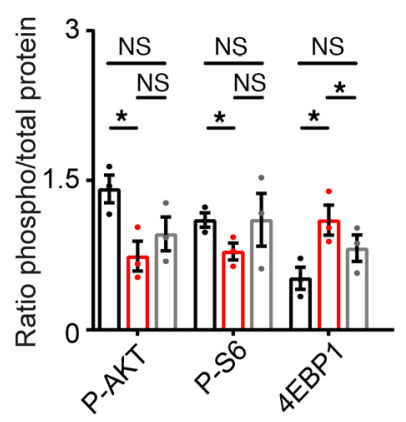

$\mathbf{F}$

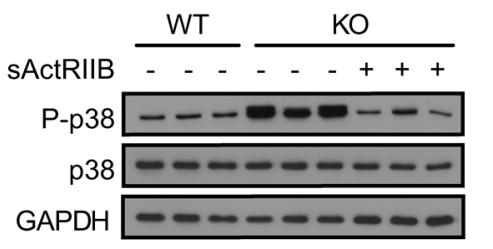

H
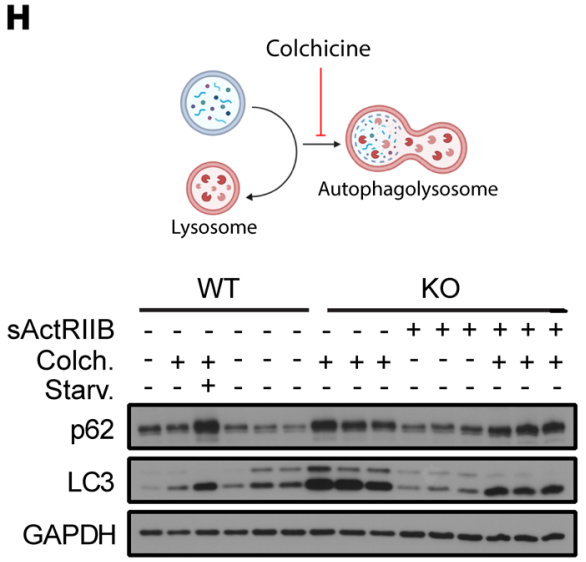

$\mathbf{E}$

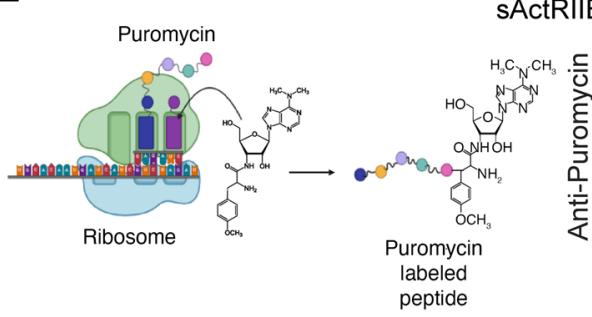

RIIB $\frac{\mathrm{WT}}{--{ }^{-}} \frac{\mathrm{KO}}{-}-+++$
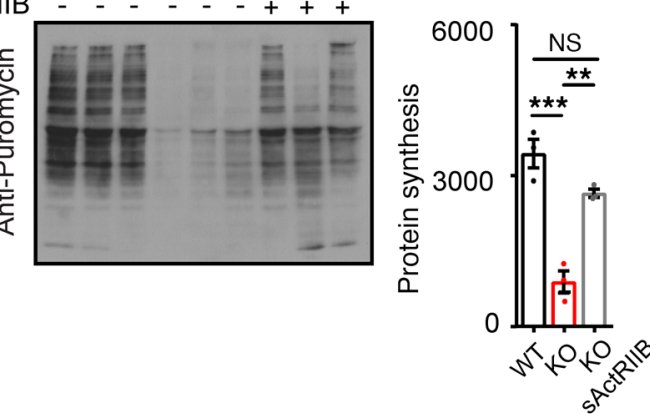

G
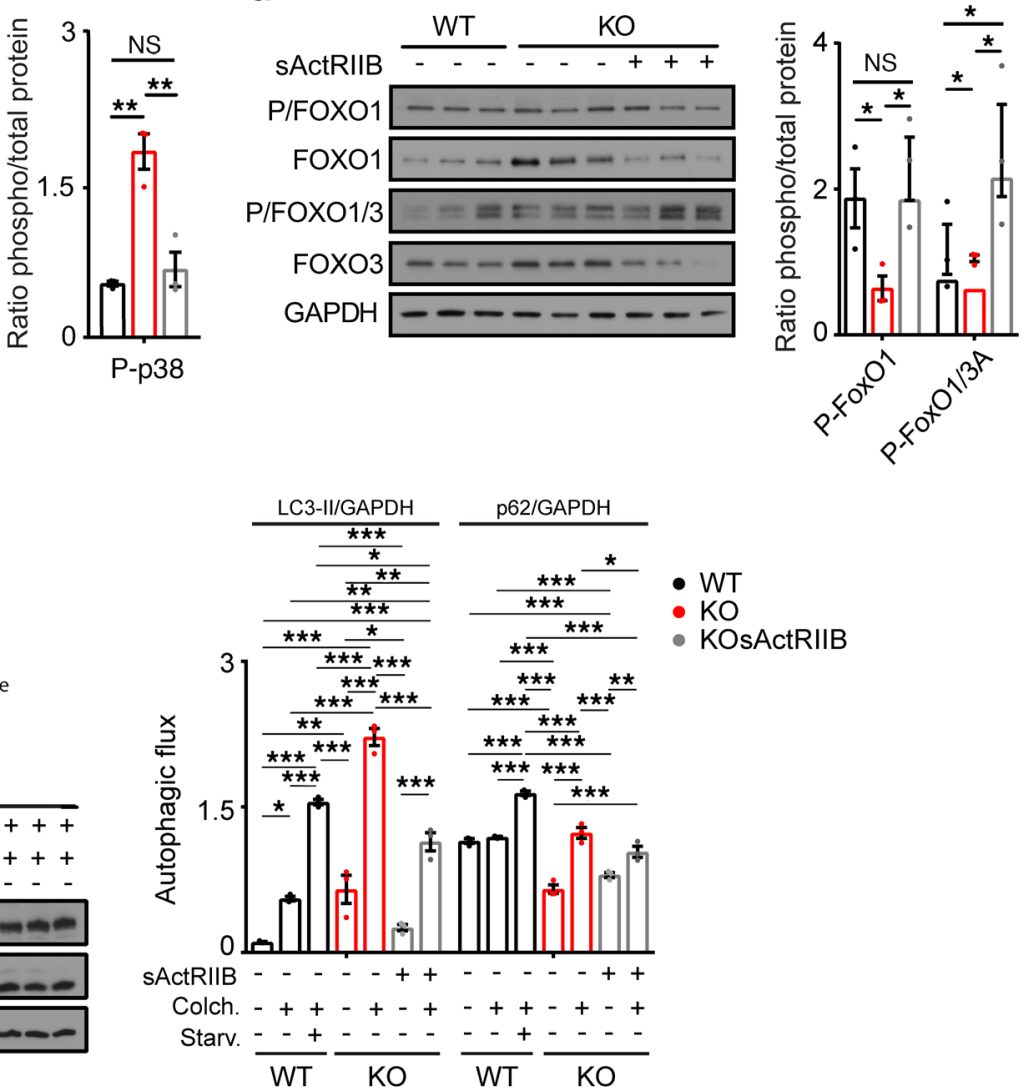
Figure 7. Pharmacological inhibition of activin restores protein synthesis while inhibiting protein breakdown. (A and B) Activin A, IL-6, and corticosterone blood level determined by ELISA in WT, Kif $3 a^{\Delta T u b}$, and Kif $3 a^{\Delta T u b}$ sActRIIB mice. A: WT $n=3, K i f 3 a^{\Delta T u b} n=5, K i f 3 a^{\Delta T u b} s A c t R I I B ~ n=5$. B left panel: WT $n=4, K i f 3 a^{\Delta \mathrm{Tub}} n=4$, Kif3a $a^{\Delta \mathrm{Tub}}$ sActRIIB $n=4$. B right panel: WT $n=10, K_{i f 3 a^{\Delta T u b}} n=8, K i f 3 a^{\Delta \text { Tub }}$ sActRIIB $n=10$. (C and D) Total protein extracts from muscle of 6-week-old WT, Kif $3 a^{\Delta T u b}$, and Kif $3 a^{\Delta T u b}$ sActRIIB mice were immunoblotted with the indicated antibodies ( $n=3$ mice). (E) Quantification of puromycin incorporation in GC muscle from 6-week-old WT Kif3 $a^{\Delta \text { Tub }}$ and Kif $3 a^{\Delta T u b}$ sActRIIB mice, using in vivo SUnSET technique ( $n=3$ mice). (F and $\mathbf{G}$ ) Total protein extracts from muscle of 6-week-old WT, Kif3 $a^{\Delta T u b}$, and Kif3 $a^{\Delta T u b}$ sActRIIB mice were immunoblotted with the indicated antibodies ( $n=3$ mice). (H) Autophagy flux analysis with colchicine treatment. Immunoblot analysis of $p 62$ and LC3 of protein extracts from GC muscles from fed and starved 6-week-old WT, Kif $3 a^{\Delta T u b}$, and $K i f 3 a^{\Delta \text { Tub }}$ sActRIIB mice $(n=3$ mice). Values are mean \pm SEM. Comparisons of more than 2 groups were calculated using 1-way ANOVA with Tukey's multiple-comparison tests. ${ }^{*} P<0.05$, ${ }^{* *} P<0.01$, ${ }^{* * *} P<0.001$. In $\mathbf{H}$, only statistically significant comparisons are shown.

tion from cyst formation (Supplemental Figure 9B), which attenuated the expected loss of kidney function (Supplemental Figure 9C).

Ultrastructural analyses revealed extensive cell death in proximal tubuli and numerous large cysts surrounded by a flat epithelium in untreated Kif3a $3 a^{\Delta T u b}$ mice, all of which were partially averted by sActRIIB (Supplemental Figure 9D). Furthermore, sActRIIB treatment prevented mitochondrial alterations (Supplemental Figure 9E), and reductions in the levels of RCC proteins, including translocase of outer membrane 20 and peroxisome proliferator-activated receptor $\gamma$ coactivator 1- $\alpha$ (Supplemental Figure 9F).

In vivo targeting of activin A signaling in muscle prevents $C K D$ induced skeletal muscle wasting. To determine whether blocking activin A signaling directly in the muscle is sufficient to rescue muscle mass in the context of ongoing experimental CKD, we used recombinant serotype 6 adeno-associated virus vectors to downregulate the activin A receptor (ACVR2A/B) in a specific muscle before the induction of AN (Figure 8A and Supplemental Figure 10A). Adenine diet led to progressive kidney disease characterized by inflammation and tubulointerstitial fibrosis (Figure 8B) and upregulation of mRNA levels of Inhba in the kidney (Figure $8 \mathrm{C}$ ). This led to increased circulating levels of activin A (Figure 8D) and loss of body weight (Figure 8E).

Muscles were analyzed 21 days after the adenine diet was started and viruses injected. Administration of (AAV-)Acvr2a/ b-shRNA resulted in a downregulation of $40 \%$ to $50 \%$ of the Acvr2a/b expression in the TA muscle of WT and AN mice (Supplemental Figure 10B) compared with those injected with (AAV-) Scramble-shRNA. Downregulation of ActRIIB/A signaling in TA muscles was sufficient to limit muscle mass loss in AN mice (Figure 8F). Differences in myofiber size between (AAV-)Acvr2a/ b-shRNA and (AAV-)Scramble-shRNA were consistent with the proportional differences in muscle mass observed in the same conditions (Figure 8G). No difference was observed in muscle weight and fiber size of WT mice upon (AAV-)Acvr2a/b-shRNA injection compared to scramble.

Together, these results supported a protective effect of muscle-specific activin A signaling downregulation, despite high levels of circulating activin A and severely affected renal function.

\section{Discussion}

The communication between different tissues is a key evolutionary feature of complex organisms (37). There are numerous examples where a disease that develops in one organ leads to the functional failure of others via a crosstalk mediated by soluble factors (12, 38-42). As CKD develops and progresses, other organs, including the liver, vasculature, lung, and heart, are also directly affected (43-45). Here, we identified, likely for the first time, a crosstalk between kidney and muscle, confirming the central role of the kidney as a master regulator of interorgan signaling (Figure 9).

Multiple studies have shown how muscle wasting is often the result of dysregulated protein synthesis, proteasomal activity, and autophagy (13). To our knowledge, this is the first time that skeletal muscle wasting has been systematically and thoroughly characterized in experimental CKD, showing that attenuated muscle growth depends on reduced rate of protein synthesis, as well as increased protein breakdown mediated by high autophagy flux. These findings were also supported by quantitative and qualitative alterations of specific muscle force, which clearly suggest functional impairment. Recent studies have shown in experimental models (46) and patients with CKD (47) that presence of muscle fibrosis correlates inversely with muscle function. While we did not observe an overt increase in muscle fibrosis to explain the loss of specific force in experimental CKD, we identified changes in the organization and density of subcellular organelles such as mitochondria, as in previous reports in aged rodents (48).

Renal interstitial fibrosis is considered the ultimate end point of all diseases leading to CKD (49), irrespective of their initial etiology, leading to irreversible loss of kidney function (50, 51). Transdifferentiation of fibroblasts/pericytes into myofibroblasts has been previously described as a key element of the profibrotic processes (52-56). Importantly, transcriptional profiles of myofibroblasts during renal fibrosis show, among the most upregulated genes, Inhba (57), which encodes the secreted protein activin A that acts both as a growth and differentiation factor $(58,59)$ and is a potent inhibitor of muscle growth $(35,60)$. Here, we showed that, both in experimental mouse models and in humans, increased production of activin A occurs specifically in kidneys in response to CKD.

Increased circulating pro-cachectic factors (e.g., activin A) have been observed in patients with cancer $(22,61)$, in patients with chronic obstructive pulmonary disease (23), and now in patients with CKD. Furthermore, our findings also suggest that blood levels of activin A increased with the progression of renal impairment. For this reason, we propose that there is a double hit effect during CKD with increased production of activin A and elevated circulating levels, which are exacerbated by impaired kidney clearance. Therefore, the inactivation of circulating pro-cachectic signals, or a direct inhibition of ActRIIB signaling directly in muscle, may be an efficient therapeutic intervention.

Indeed, our data show that a pharmacological approach, using the sActRIIB, successfully prevented muscle loss in experimental CKD, normalizing muscle force as well as mitochondrial mass, localization, and function. This was also confirmed by the normalization of protein synthesis and the expression of genes regulating proteasome-mediated protein degradation and autophagy. Administration of soluble ActRIIA/B blocking antibodies 

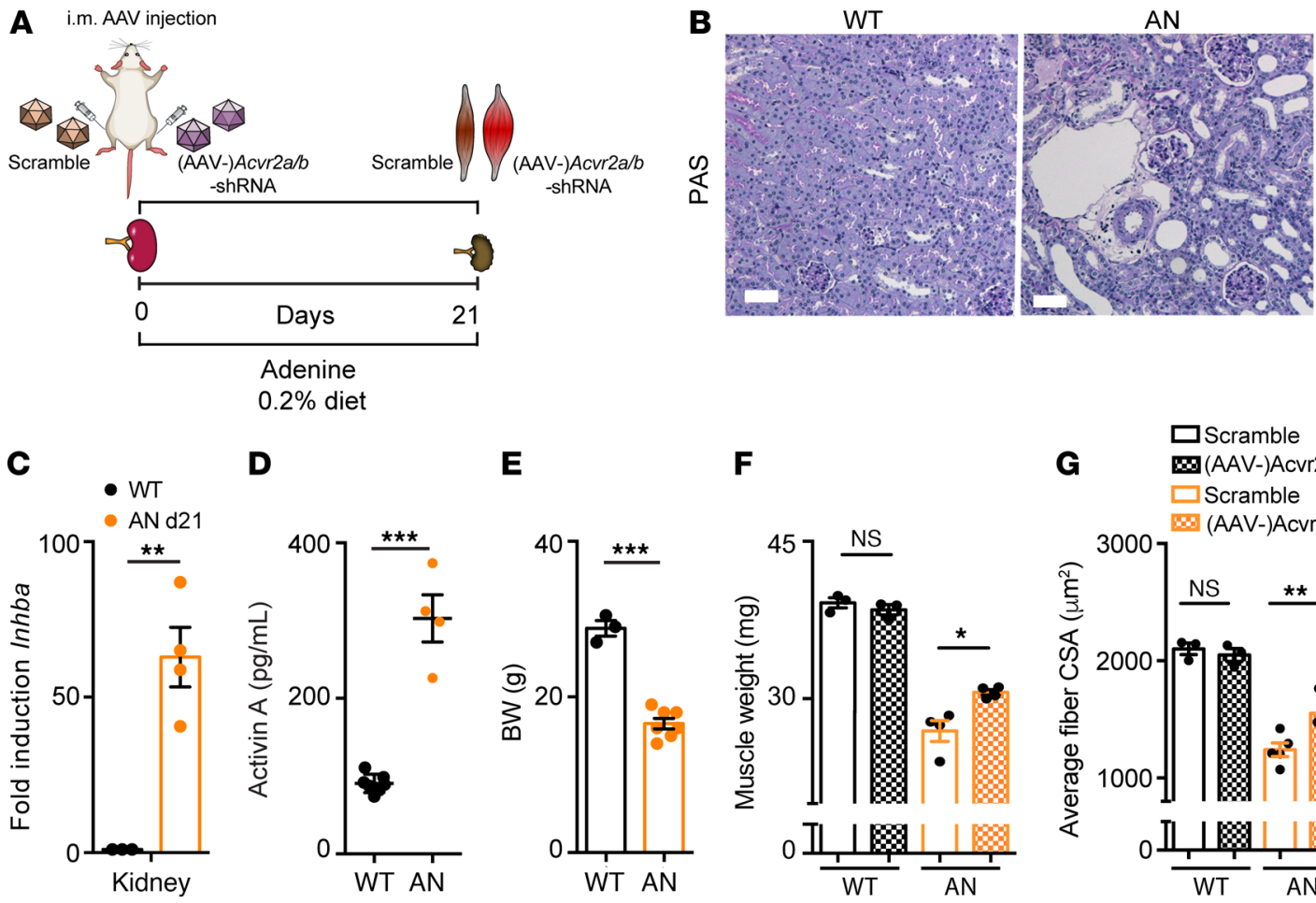

D

E

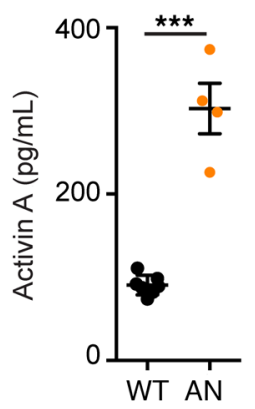

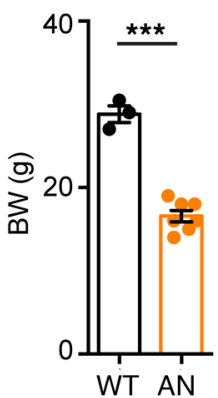

$\mathbf{F}$

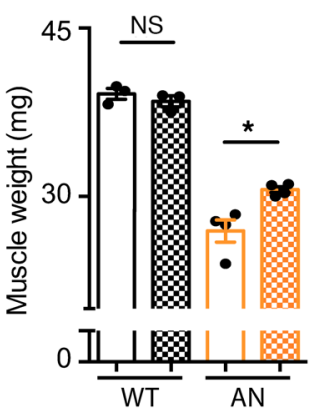

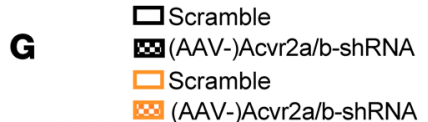

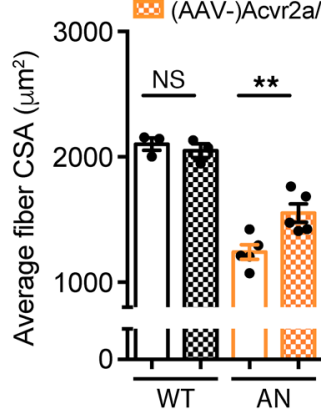

Figure 8. Muscle-specific downregulation of activin pathway protects from CKD-induced skeletal muscle atrophy. (A) Schematic representation of experimental design: mice were fed with adenine-enriched diet to induce 2,8-DHA nephropathy (AN), and TA muscles were transfected with (AAV-) Acvr2a/b-shRNA or (AAV-)Scramble-shRNA. Muscles were examined 3 weeks later. (B) Representative images of the renal histology of WT and AN kidney. Scale bars represent $60 \mu \mathrm{m}$. PAS, periodic acid-Schiff. (C) Quantitative RT-PCR analysis of Inhba in kidneys of WT and AN mice ( $n=4$ mice). (D) Blood levels of activin A in WT and AN mice determined by ELISA $(n=4$ mice). (E) Body weights were measured in WT and AN mice $(n=7$ mice). (F) The weight of (AAV-)Acvr2a/b-shRNA- and (AAV-)Scramble-shRNA-infected muscles of WT and AN mice were measured ( $n=4$ mice). (C) Average fiber crosssectional area of TA muscle of WT and AN mice infected with (AAV-)Acvr2a/b-shRNA or (AAV-)Scramble-shRNA ( $n=3$ mice). Data shown as mean \pm SEM. Student's $t$ test used for statistical significance. ${ }^{*} P<0.05,{ }^{* *} P<0.01,{ }^{* *} P<0.001$.

has already been used in preventing muscle wasting in different mouse models of glucocorticoid-induced atrophy (62), of microgravity (63), and of sarcopenia (64) and in cancer-associated cachexia $(61,65)$.

Based on these findings, activin A blockade could be considered as a potential clinical pharmacological intervention in CKD patients with skeletal muscle wasting. While different soluble receptor ligand traps are being actively tested in various clinical trials $(66,67)$, broad tissue expression of ActRIIB and its promiscuous TGF- $\beta$ superfamily ligands reduce the interest in using ligand-trapping approaches directed at ActRIIB (68). Indeed, we showed that ligand trap treatment has a direct effect in the attenuation of kidney pathology. Given that kidney function affects the levels of circulating factors (i.e., loss of renal function may lead to an accumulation in blood) - then improving renal function could simply further reduce circulating levels of activin A, which should also prevent muscle wasting. For this reason, we considered a different approach based on viral targeting. We demonstrated that attenuating ActRIIB/A intramuscular signaling with a tissue-specific (AAV-)Acvr2a/b-shRNA is an effective tool to reduce muscle wasting associated with $\mathrm{CKD}$. In addition, we provide data in control mice, showing no difference in muscle weight and cross-sectional area between those injected with Scramble or Actr $2 a / b$-shRNA, thus ruling out the possibility of compensatory mechanisms inducing hypertrophy, which could blur our conclusions. Furthermore, the amelioration of muscle wasting was independent of severe kidney disease and occurred despite elevated circulating levels of pro-cachectic ligands. Thus, these results demonstrate the utility of our approach for dissociating such ligands from their effects on intracellular signaling processes that promote muscle wasting.

To our knowledge, this is the first study to investigate the effect of the downregulation of both Acvr2b and Acvr2a in adult mice. Indeed, a recent study (69) showed how targeting the Acvr2b and Acvr2a in muscle leads to muscle hypertrophy. In this paper, the authors used a myosin light chain promoter/enhancer that leads to the deletion of the Acvr2b and Acvr2a from E13 days, which may have impacted muscle development from an embryonic stage. In contrast, we used a system where we downregulate the Acvr2b and Acvr2a in the muscle of adult mice, excluding the possibility of compensatory hypertrophy. We propose that similar strategies could be engineered in the future in order to limit the risk of off-target effects.

Muscle wasting in CKD is the consequence of a complex interplay of different factors that induces systemic and multiorgan complication (70). We hypothesize that, while the initial source of the pro-cachectic and proinflammatory molecules is the kidney, other organs play a critical role in regulating the anabolic/ 


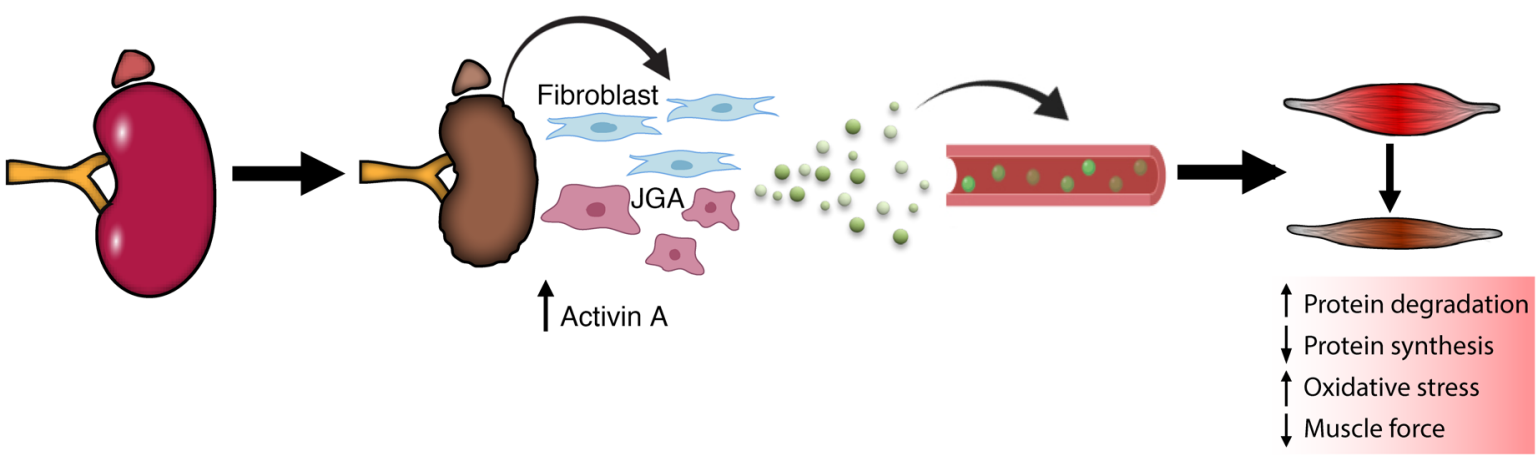

Figure 9. Schematic overview of proposed crosstalk between kidney and muscle during CKD. We propose a new kidney/muscle axis where the soluble pro-cachectic factor activin A is produced in injured kidney, accumulates in the blood, and destroys muscle homeostasis and induces muscle wasting.

catabolic balance in the muscle. In this context, the central nervous system might contribute to the integration of inflammatory signals by increasing the circulating levels of glucocorticoids (71). Furthermore, the accumulation of uremic toxins in the body under CKD could contribute to muscle loss $(72,73)$. However, our study shows that the muscle wasting actions of other pro-cachectic cytokines (IL-6, corticosterone) are overtaken by the modulation of activin A, suggesting that activin A signaling is a dominant step in CKD-induced cachexia.

In conclusion, we propose a model where soluble procachectic factors accumulated in the blood in response to kidney injury, disrupting homeostasis and inducing skeletal muscle wasting. This process can be prevented by attenuating activin A signaling, which offers the potential to effectively prevent $\mathrm{CKD}$-induced muscle wasting.

\section{Methods}

Animals and treatment. All animals were housed in a specific pathogen-free facility with free access to chow and water and a 12-hour light/12-hour dark cycle. Breeding and genotyping were done according to standard procedures. Kif $3 a^{\mathrm{fl} / \mathrm{fl}}$ mice were crossed to $K s p^{\text {Cre }}$ mice to generate Kif $3 a^{\Delta \mathrm{Tub}}$ mice (24).

Postnatal myostatin/activin block was induced in 2-week-old male mice, through intraperitoneal (i.p.) injection with $10 \mathrm{mg} / \mathrm{kg}$ of sActRIIB-Fc every week, 2 times till week 6. Blood samples were drawn by puncturing the right retro-orbital plexus under isoflurane anesthesia. BUN was measured using urea kits (Lehmann) following the manufacturer's instructions. Blood was collected into EDTA tubes for ELISA. Activin A (R\&D Systems, Bio-Techne; DACOOB), IL-6 (R\&D Systems, Bio-Techne; MTA00B), corticosterone (Abcam; ab108821), and GDF8 (R\&D Systems, Bio-Techne; DGDF80) were measured following the manufacturers' instructions.

Mouse model of 2,8-DHA nephropathy. We induced 2,8-DHA nephropathy in 12 -week-old male mice by administration of a $0.2 \%$ adenine-containing chow (Altromin) $(29,30)$.

Construction of the ACVR2A and ACVR2B shRNA and AAV vector production. ACVR2A- and ACVR2B-targeting shRNAs were designed using the GPP Web Portal (https://portals.broadinstitute.org/gpp/ public/), and AAV-shRNA-ctrl (Addgene plasmid 85741) was used as a backbone and control vector. shRNA oligonucleotides were subcloned into BamHI and XbaI sites of AAV-shRNA-ctrl to generate AAV-shRNA-Acvr2 $a$ and AAV-shRNA-Acvr $2 b$ vectors. We used
Acvr2a-shRNA (shRNA1 from knockdown efficiency validation) forward primer: 5'-GATCCGCTAGAGGATTGGCATATTTACTCGAGTAAATATGCCAATCCTCTAGCTTTTTT-3'; Acvr2a-shRNA reverse primer: 5 '-CTAGAAAAAAGCTAGAGGATTGGCATATTTACTCGAGTAAATATGCCAATCCTCTAGCG-3'; Acvr2b-shRNA forward primer: 5'-GATCCGGGAGTGCATCTACTACAACGCTCGAGCGTTGTAGTAGATGCACTCCCTTTTTT-3'; Acvr2b-shRNA (shRNA1 from knockdown efficiency validation) reverse primer: 5' -CTAGAAAAAAGGGAGTGCATCTACTACAACGCTCGAGCGTTGTAGTAGATGCACTCCCG-3'.

A total of $6 \times 10^{5} \mathrm{HEK} 293 \mathrm{~T} / 17$ cells (ATCC CRL-11268) were seeded in each well of 6-well plates and incubated overnight at $37^{\circ} \mathrm{C}$ in a humidified incubator containing $5 \% \mathrm{CO}_{2}$ to achieve approximately $70 \%$ to $80 \%$ confluence. Each well was cotransfected by $0.5 \mu$ g plasmid encoding ACVR2A-HA (Addgene plasmid 11754) or ACVR2B-MYC, which was generated by modification of ACVR2B (Addgene plasmid 13501) with a MYC tag before the stop codon of the ACVR2B gene, and $1.5 \mu \mathrm{g}$ plasmids expressing either shRNA control or different shRNA using PolyFect Transfection Reagent (QIAGEN), following the manufacturer's instructions. The transfected cells were incubated at $37^{\circ} \mathrm{C}$ for 24 hours before harvesting for protein analysis.

AAVs were produced by cotransfection of HEK293T/17 cells (ATCC CRL-11268) with AAV-shRNA vectors and the packaging/ helper plasmid pDGM6 (Addgene, plasmid 110660), which includes the AAV2 rep genes, AAV6 cap genes, and adenovirus helper genes (74). Three days after transfection, cell lysates were subjected to freeze-thaw cycles in PBS-MK $\left(1 \times \mathrm{PBS}, 1 \mathrm{mM} \mathrm{MgCl}_{2}, 2.5 \mathrm{mM} \mathrm{KCl}\right)$, and the vectors were purified by iodixanol gradient ultracentrifugation followed by dialysis and concentration against PBS using centrifugal filters (Sartorius Vivaspin 20 Centrifugal Concentrators, PES Membrane 10,000 Da). Physical particles were quantified by real-time PCR (75), and titers are expressed as viral genomes per milliliter $(\mathrm{vg} / \mathrm{mL})$.

$A A V$ injection. TA muscles of 3-month-old $\mathrm{BALB} / \mathrm{c}$ mice were injected with (AAV-)Acvr $2 a / b$-shRNA while contralateral muscles received (AAV-)Scramble-shRNA, and mice were fed with adenineenriched diet. ( $n=4$ for each condition.)

(AAV-)Acvr2a-shRNA and (AAV-)Acvr2b-shRNA were mixed with $5 \times 10^{9} \mathrm{vg}$ from each and used at $10 \times 10^{9} \mathrm{vg}$ in total; (AAV-) Scramble-shRNA was used at $10 \times 10^{9} \mathrm{vg}$.

Transcriptome analysis. RNA was purified using standard procedures and analyzed using Affymetrix Mouse Genome 4302.0 gene chips. Raw data were obtained by standard array hybridization tech- 
niques and normalized via the Single Channel Array Normalization algorithm (76) mapping the probes to the custom chip definition file from the Brainarray resources in v18.

Raw mapped reads were used to determine differentially expressed genes and generate normalized read counts to visualize as heatmaps using Morpheus (Broad Institute).

Microarray data on mouse kidney and muscle were deposited in the National Center for Biotechnology Information's Gene Expression Omnibus database (GSE169316).

Real-time quantitative PCR. RNA was extracted using QIAzol lysis reagent (QIAGEN) according to manufacturer instructions. RNA to cDNA conversion was performed using the High Capacity cDNA Reverse Transcription Kit (Applied Biosystems, Thermo Fisher Scientific). Quantification of target genes was done by quantitative PCR using TaqMan technology (Applied Biosystems, Thermo Fisher Scientific). Reaction mixes were run on the QuantStudio 3 (Applied Biosystems, Thermo Fisher Scientific) thermal cycler. TaqMan primer pairs used to quantify target genes were as follows: Inhba: Mm00434338_m1; Actb: Mm02619580_g1; Acvr2a: Mm01331097_m1; Acvr2b: Mm00431664_m1; Gapdh: Mm99999915_g1 (Thermo Fisher Scientific).

Kidney tissue profiling from human samples. For bulk mRNA processing, glomerular expression profiles from microdissected kidney tissue were generated from participants with biopsy-proven FSGS enrolled in NEPTUNE study. RNA-Seq expression data were obtained from 66 patients with FSGS (77). Briefly, Illumina TruSeq mRNA sample prep v2 kit was used to process the mRNA samples. Samples were run on Illumina HiSeq2000, paired-end read length of 100 bases at the facility of University of Michigan Advanced Genomics Core (https:// brcf. medicine.umich.edu/cores/advanced-genomics/). Quality of the data was assessed using the standard measures using FASTQC tool. Annotation and quantification of mapping results on gene level were performed using HTseq (78) and transformed with voom (78). All downstream analyses were performed using the voom-transformed intensities.

Single-cell expression profiling. The Kidney Precision Medicine Project (KPMP) adult human kidney reference data set was created by the combined analysis of 24 scRNA-Seq data sets, including 16 tumor nephrectomies, 5 surveillance, and 3 living donor tissue biopsies (77). Tissue dissociation into single-cell suspensions was accomplished by the enzyme Liberase TL (Roche, 5401020001) at $37^{\circ} \mathrm{C}$ for 12 minutes. $10 \mathrm{x}$ Genomics technology was used to generate the single-cell sequencing data. Clustering and the downstream analysis of the sequencing data was performed using Seurat (version3) R package (79).

Human microarray analysis. Human renal biopsy specimens and Affymetrix microarray expression data were obtained within the framework of the European Renal cDNA Bank-Kröner-Fresenius Biopsy Bank (80). Published data sets of glomerular and tubulointerstitial samples from patients with different CKDs were analyzed for mRNA expression levels. Analysis included gene expression profiles from patients with cadaveric donor, tumor nephrectomy, Antineutrophil cytoplasmic antibody-associated glomerulonephritis, lupus nephritis, membranous nephropathy, FSGS, IgA nephropathy, diabetic nephropathy, hypertensive nephropathy, minimal change disease, and controls (living donors; GSE99340, GSE32591, GSE35489, GSE37463). Grouping of the patients into different CKD stages (CKD 1-5) was done as published (28). CEL file normalization was per- formed with the Robust Multichip Average method using RMAExpress (Version 1.0.5) and the human Entrez Gene custom CDF annotation from Brainarray version 18 (http://brainarray.mbni.med.umich.edu/ Brainarray/Database/CustomCDF/genomic_curated_CDF.asp). To identify differentially expressed genes, the Significance Analysis of Microarrays (SAM) method (81) was applied using SAM function in Multiple Experiment Viewer (TiGR MeV, Version 4.9). Correlation of eGFR calculated by the CKD Epidemiology Collaboration equation (82) was computed with the log-transformed steady-state expression levels of glomerular gene expression profiles from patients with different CKDs.

Single-cell analysis of Kif $3 a^{4 T u b}$ and control kidneys. A single-cell suspension was prepared using a cold active protease (CAP) as described in previous studies (77). Briefly, 1 kidney per mouse was minced into pieces on ice, transferred to 2 hard tissue-homogenizing tubes (CK28, Precellys, Bertin Instruments) filled with $1 \mathrm{~mL}$ ice-cold PBS, and homogenized using Minilys (Bertin Instruments) at the highest speed for 15 seconds. The homogenized kidney was centrifuged at $400 \mathrm{~g}$ for 4 minutes at $4^{\circ} \mathrm{C}$. The kidney pellet was suspended in $2 \mathrm{~mL}$ red blood lysis buffer (R7757, MilliporeSigma) for 2 minutes to remove red blood cells. After spin down the kidney pellet was suspended in $2 \mathrm{~mL}$ CAP solution $(10 \mathrm{mg} / \mathrm{mL}$ Bacillus licheniformis protease [P5380, MilliporeSigma], $5 \mathrm{mM} \mathrm{CaCl}_{2}, 100 \mathrm{U} / \mathrm{mL}$ DNase [04536282001, Roche] in PBS) for 20 minutes on ice with repeated trituration steps for 20 seconds every 5 minutes. The digestion was neutralized by $15 \mathrm{~mL}$ PBS supplemented with $10 \%$ fetal bovine serum and passed through a $40 \mu \mathrm{m}$ Corning cell strainer. Cells were centrifuged at $400 \mathrm{~g}$ for 4 minutes at $4^{\circ} \mathrm{C}$ and washed twice in $20 \mathrm{~mL}$ PBS supplemented with $0.5 \%$ bovine serum albumin. Dead cells were removed by Dead Cell Removal Kit (130-090-101, Miltenyi Biotec) according to the manufacturer's protocol. The living cells were passed through a $30 \mu \mathrm{m}$ cell strainer (04-004-2326, Sysmex), and cell concentration was determined using a TC2O automated cell counter (Bio-Rad). Cells were loaded on a 10x Genomics Chromium single-cell instrument. All steps were performed according to standard protocol of the Chromium single-cell 3' v3 kit to generate high-quality cDNA libraries.

Preprocessing and quality control of scRNA-Seq data. Raw sequencing data from 10x Genomics were processed using CellRanger software (version 3.0.2, 10x Genomics) and the 10x mouse genome mm10 3.0.0 release as the reference (function cellranger count). The matrices of cells and the unique molecular identifier (UMI) count were obtained and further processed by the R package Seurat (version 3.1.1; ref. 83). As a quality control (QC) step, we first filtered out genes detected in fewer than 3 cells and those cells in which fewer than 200 genes had nonzero counts. To remove potential doublets, cutoff values for number of expressed genes (nGene) were determined for samples following visual inspection of the distribution of nGene and number of UMIs for each cell. The cells with higher nGene values (i.e., 6000) were excluded. We further removed low-quality cells with more than $50 \%$ mitochondrial genes of all detected genes (84).

Dimensionality reduction and clustering. The Seurat $\mathrm{R}$ package (version 3.1.1) was used to perform unsupervised clustering analysis on scRNA-Seq data. In brief, gene counts for cells that passed QC were normalized by library size and log-transformed (function NormalizeData, normalization.method = "LogNormalize," scale. factor $=10,000$ ). Then, highly variable genes were detected (function FindVariableFeatures, selection.method = "vst," nfeatures 
$=2000$ ). To reduce the batch effects, we applied the integration method implemented in the latest Seurat v3 (function FindIntegrationAnchors and IntegrateData, dims $=1: 30$ ). The integrated matrix was then scaled by ScaleData (default parameters). Principal component (PC) analysis was performed on the scaled data (function RunPCA, npcs $=30$ ) in order to reduce dimensionality. The number of PCs used for each clustering round was data set dependent, and they were estimated by the elbow of a principal component analysis scree plot. The selected PCs were then used to compute the K-nearest neighbors graph based on the Euclidean distance (function FindNeighbors), which was later used to generate cell clusters using function FindClusters. The resolution of FindClusters function for each data set was determined (0.6) by exploration of top marker genes of each cluster. t-SNE was used to visualize clustering results. The top differentially expressed genes in each cluster were found using the FindAllMarkers function (min.pct $=0.25$, logfc.threshold $=0.25$ ) that ran Wilcoxon's rank-sum tests. The top expressed genes were then used to determine the cell type of each cluster. Data availability: Gene Expression Omnibus GSE172252.

Morphological analysis of kidney and muscle sections. Mouse kidneys were perfusion fixed in $4 \%$ phosphate-buffered paraformaldehyde, embedded in paraffin, and further processed for period acidSchiff and H\&E stainings. Slices were analyzed using an inverted fluorescence microscope (Zeiss).

RNAscope in situ hybridization. FISH was performed in formalinfixed, paraffin-embedded murine kidney samples using RNAscope technology as previously described (85). The RNAscope MmInhba-probe from Advanced Cell Diagnostics (catalog 455871) was used as the target probe to detect Inhba mRNA. Fluorescent labeling of the target probe was performed using OPAL 690 dye from Akoya Biosciences (catalog FP1497001KT, dilution 1:1000). Nuclear costaining was performed using the DAPI solution from Advanced Cell Diagnostics. Fluorescence imaging was performed using the THUNDER Imager (Leica Microsystems).

SDH staining of muscle sections. Muscle cryosections were incubated for 3 minutes at room temperature in a sodium phosphate buffer containing $75 \mathrm{mM}$ sodium succinate, $1.1 \mathrm{mM}$ Nitroblue Tetrazolium (MilliporeSigma), and $1.03 \mathrm{mM}$ Phenazine Methosulphate (MilliporeSigma). Samples were then fixed in $10 \%$ formal-calcium, dehydrated, and cleared in xylene prior to mounting with DPX mounting medium (Thermo Fisher Scientific).

Immunofluorescence staining of kidney sections. Cryosections, $4 \mu \mathrm{m}$, were incubated with antibodies listed in Supplemental Table 1. Hoechst costaining allowed us to identify the SS position of myonuclei. Imaging was performed with a laser-scanning microscope (Zeiss LSM 800).

Western blot. Muscles were lysed and immunoblotted as previously described (86). Kidneys were homogenized in lysis buffer (containing $20 \mathrm{mM}$ CHAPS and $1 \%$ Triton X-100). After centrifugation $\left(15,000 \mathrm{~g}, 15\right.$ minutes, $\left.4^{\circ} \mathrm{C}\right)$ protein concentration was determined by Bio-Rad protein assay. Equal amounts of protein were separated on SDS-PAGE. Blots were stripped using Restore Western Blot Stripping Buffer (Pierce, Thermo Fisher Scientific) according to the manufacturer's instructions and probed again when necessary. The antibodies used are listed in Supplemental Table 1. Uncropped images of blots are shown in the supplementaty material.

Muscle tension measurements. Dissection of the hind limb was carried out under oxygenated Krebs solution $\left(95 \% \mathrm{O}_{2}\right.$ and $\left.5 \% \mathrm{CO}_{2}\right)$.
Under circulating oxygenated Krebs solution, one end of a silk suture was attached to the distal tendon of the EDL and the other to a force transducer (FTO3, Grass Instruments). The proximal tendon remained attached to the tibial bone. The leg was secured in the experimental chamber. Silver electrodes were positioned on either side of the EDL. A constant voltage stimulator was used to directly stimulate the EDL, which was stretched to attain the optimal muscle length to produce maximum twitch tension. Tetanic contractions were invoked by stimulus trains of $500 \mathrm{~ms}$ duration at 20,50,100, and $200 \mathrm{~Hz}$. The maximum tetanic tension was determined from the plateau of the frequency-tension curve.

Transmission electron microscopy. Biceps muscle and the kidney were briefly fixed with $4 \%$ paraformaldehyde and $2.5 \%$ glutaraldehyde in $0.1 \mathrm{M}$ cacodylate buffer $\mathrm{pH} 7.4$ in situ at room temperature (RT), then dissected, removed, and cut into pieces of $1 \mathrm{~mm}^{3}$ and fixed for 48 hours in the same solution at $4^{\circ} \mathrm{C}$. Tissue blocks were contrasted using $1 \% \mathrm{OsO}_{4}$ (Roth; RT, 1 hour) and $1 \%$ uranyl acetate (Polysciences) in $70 \%$ ethanol (RT, 1 hour). After dehydration, tissue blocks were embedded in epoxy resin (Durcopan), and ultrathin sections of 50 $\mathrm{nm}$ thickness were cut using a Leica EM UC6 ultramicrotome (Leica Microsystems). Sections were imaged using a Zeiss 910 Transmission Electron Microscope and analyzed using ITEM software.

Autophagic flux quantification. Autophagic flux was monitored in fed condition using colchicine (C9754, MilliporeSigma) as previously described (26). Briefly mice were treated, by i.p. injection, with vehicle or with $0.4 \mathrm{mg} / \mathrm{kg}$ colchicine. The treatment was administered twice, at 24 hours and at 12 hours before muscle collection.

In vivo protein synthesis measurements. In vivo protein synthesis was measured by using the SUnSET technique (25). Mice were anesthetized and then given an i.p. injection of $0.040 \mu \mathrm{mol} / \mathrm{g}$ puromycin dissolved in $100 \mu \mathrm{L}$ of PBS. At exactly 30 minutes after injection, muscles were collected and frozen in liquid $\mathrm{N}_{2}$ for Western blot analysis.

Statistics. All statistical analyses were performed using GraphPad Prism 8 and IBM SPSS (v25). Results are reported as mean \pm SEM or median \pm IQR. To identify differentially expressed genes, the SAM method (81) was applied using SAM function in Multiple Experiment Viewer (TiGR MeV, Version 4.9). Comparisons of more than 2 groups were calculated using 1-way ANOVA with Tukey's multiple-comparison tests. Correlation analyses were performed using Spearman's correlation coefficient. Significant differences between 2 groups were performed by the 2-tailed Student's $t$ test for independent variables. Differences between groups were considered statistically significant for Por $q<0.05$.

Study approval. Animal experiments were conducted according to the Guide for the Care and Use of Laboratory Animals (NIH; National Academies Press, 2011), as well as the German law for the welfare of animals. All animal experiments were approved by Regierungspraesidium Freiburg (G-15/143), Freiburg, Germany, and by the Italian Ministero della Salute, Ufficio VI (Rome, Italy; authorization number 1060/2015 PR).

Serum samples from healthy controls and patients with CKD were collected in accordance with approved protocols by the local ethics committee of the Chamber of Physicians in Hamburg (Germany, PV4780 and PV4806) and conducted in accordance with the ethical principles stated by the Declaration of Helsinki. Informed consent was obtained from all patients. 


\section{Author contributions}

FS, VGP, CDC, PLT, MK, MS, KP, and TBH conceived the study. FS and KP developed methodology. VGP and KP validated data. $\mathrm{EH}$, JET, JSW, OR, A Pasternack, PB, TO, and TBH provided resources. FS, OK, VGP, KP, and TBH performed formal analysis. FS, MTL, CT, MTL, S Lu, GW, S Liu YZ, R Mitchell, A Paolini CM, TK, SO, $\mathrm{AM}, \mathrm{DK}, \mathrm{VN}, \mathrm{FE}, \mathrm{R}$ Menon, IVM, BMK, and OK investigated. FS, VGP, KP, and TBH wrote the manuscript. FS, VGP, NW, KP, and OK visualized the data. VGP, KP, and TBH supervised the study. The sequence of co-senior authors was decided based on reverse alphabetical order.

\section{Acknowledgments}

We would like to thank Barbara Joch for her excellent technical support concerning the electron microscopy work. The European Renal cDNA Bank-Kröner-Fresenius Biopsy Bank (ERCB-KFB) was supported by the Else Kröner-Fresenius Foundation. We also thank all participating centers of the ERCB-KFB and their patients for their cooperation (28). The objectives and study design of NEPTUNE can be found in the ClinicalTrials.gov database under NCT1209000. All samples used in this study were obtained with patient consent and with the approval of IRBs of participating institutions. NEPTUNE data were obtained based on the multisite, central IRB approval for the study, HUM00158219, by the IRB of the University of Michigan Medical School. This study was supported, in part, by the George M. O'Brien Michigan Kidney Translational Core Center, funded by NIH/National Institute of Diabetes and Digestive and Kidney Diseases (NIDDK) grant 2P30-DK-081943. The human kidney single-cell data sets were generated by KPMP, funded by the following grants from the NIH/NIDDK: U2C DK114886, UH3DK114861, UH3DK114866, UH3DK114870, UH3DK114908, UH3DK114915, UH3DK114926, UH3DK114907, UH3DK114920, UH3DK114923, UH3DK114933, and UH3DK114937. EH was supported by the
Deutsche Forschungsgemeinschaft (DFG; Heisenberg Programme and CRC1192). TO was supported by the DFG (OS 196/4-1). PB was supported by the DFG (project numbers: 322900939, 432698239, and 454024652), the German Federal Ministry of Education and Research (BMBF: STOPFSGS-01GM1901A), and the European Research Council (ERC) under the European Union's Horizon 2020 research and innovation program (grant 101001791). MS was supported by Agenzia Spaziale Italiana (MARS-PRE) and Associazione Italiana Ricerca sul Cancro (grant 23257). OK was supported by DFG (KR1984/4-1). VGP was supported by the DFG (CRC1192), Deutsche Gesellschaft für Nephrologie, and eMed Consortia "Fibromap" (BMBF). TBH was supported by the DFG (CRC1192, HU 1016/8-2, HU 1016/11-1, HU 1016/ 12-1), by the BMBF (STOP-FSGS-01GM1901C and NephrESA-031L0191E), by the Else-Kröner Fresenius Foundation (Else KrönerPromotionskolleg-iPRIME), by the ERC (grant 616891), and by the Horizon 2020-Innovative Medicines Initiative2 consortium Biomarker Enterprise to Attack Diabetic Kidney Disease (115974); this joint undertaking receives support from the European Union's Horizon 2020 research and innovation program and European Federation of Pharmaceutical Industries and Associations and Juvenile Diabetes Research Foundation. The content is solely the responsibility of the authors and does not necessarily represent the official views of the NIH.

Address correspondence to: Tobias B. Huber or Victor G. Puelles, III. Department of Medicine, University Medical Center Hamburg-Eppendorf, Martinistraße 52, Gebäude O10, 20246 Hamburg, Germany. Phone: 49.40.741053908; Email: t.huber@uke.de (TBH); Email: v.puelles@uke.de (VGP). Or to: Ketan Patel, School of Biological Sciences, University of Reading, Reading Berkshire RG6 6AJ, United Kingdom. Phone: 44.118.378.8079; Email: ketan. patel@reading.ac.uk.
1. Collins AJ, et al. Chronic kidney disease awareness, screening and prevention: rationale for the design of a public education program. Nephrology (Carlton). 2010;15(suppl 2):37-42.

2. Levin A, et al. Global kidney health 2017 and beyond: a roadmap for closing gaps in care, research, and policy. Lancet. 2017;390(10105):1888-1917.

3. Mak RH, et al. Wasting in chronic kidney disease. JCachexia Sarcopenia Muscle. 2011;2(1):9-25.

4. Kosmadakis GC, et al. Physical exercise in patients with severe kidney disease. Nephron Clin Pract. 2010;115(1):c7-c16.

5. Sullivan DH, et al. Body weight change and mortality in a cohort of elderly patients recently discharged from the hospital. J Am Geriatr Soc. 2004;52(10):1696-1701.

6. Stenvinkel P, et al. Muscle wasting in end-stage renal disease promulgates premature death: established, emerging and potential novel treatment strategies. Nephrol Dial Transplant. 2016;31(7):1070-1077.

7. Oliveira EA, et al. Cachexia/protein energy wasting syndrome in CKD: causation and treatment. Semin Dial. 2019;32(6):493-499.

8. Peart WS. The kidney as an endocrine organ.
Lancet. 1977;2(8037):543-548.

9. Shih HM, et al. Physiology and pathophysiology of renal erythropoietin-producing cells. J Formos Med Assoc. 2018;117(11):955-963.

10. Agapova OA, et al. Ligand trap for the activin type IIA receptor protects against vascular disease and renal fibrosis in mice with chronic kidney disease. Kidney Int. 2016;89(6):1231-1243.

11. Thomas R, et al. Chronic kidney disease and its complications. Prim Care. 2008;35(2):329-344.

12. Doi K, et al. Lung injury following acute kidney injury: kidney-lung crosstalk. Clin Exp Nephrol. 2011;15(4):464-470.

13. Glass DJ. Signaling pathways perturbing muscle mass. Curr Opin Clin Nutr Metab Care. 2010;13(3):225-229.

14. Fearon KC, et al. Cancer cachexia: mediators, signaling, and metabolic pathways. Cell Metab. 2012;16(2):153-166.

15. Wang XH, Mitch WE. Mechanisms of muscle wasting in chronic kidney disease. Nat Rev Nephrol. 2014;10(9):504-516.

16. Cohen S, et al. Muscle wasting in disease: molecular mechanisms and promising therapies. Nat Rev Drug Discov. 2015;14(1):58-74.

17. Schiaffino S, et al. Mechanisms regulating skeletal muscle growth and atrophy. FEBS J. 2013;280(17):4294-4314.

18. Zimmers TA, et al. Induction of cachexia in mice by systemically administered myostatin. Science. 2002;296(5572):1486-1488.

19. Chen JL, et al. Elevated expression of activins promotes muscle wasting and cachexia. FASEBJ. 2014;28(4):1711-1723.

20. von Haehling S, et al. Muscle wasting and cachexia in heart failure: mechanisms and therapies. $\mathrm{Nat}$ Rev Cardiol. 2017;14(6):323-341.

21. Anker SD, Sharma R. The syndrome of cardiac cachexia. Int J Cardiol. 2002;85(1):51-66.

22. Loumaye A, et al. Role of Activin A and myostatin in human cancer cachexia. JClin Endocrinol Metab. 2015;100(5):2030-2038.

23. Zhou G, et al. Elevated serum Activin A in chronic obstructive pulmonary disease with skeletal muscle wasting. Clinics (Sao Paulo). 2019;74:e981.

24. Lin F, et al. Kidney-specific inactivation of the KIF3A subunit of kinesin-II inhibits renal ciliogenesis and produces polycystic kidney disease. Proc Natl Acad Sci U S A. 2003;100(9):5286-5291.

25. Schmidt EK, et al. SUnSET, a nonradioactive method to monitor protein synthesis. Nat Methods. 2009;6(4):275-277. 
26. Milan G, et al. Regulation of autophagy and the ubiquitin-proteasome system by the FoxO transcriptional network during muscle atrophy. Nat Commun. 2015;6:6670.

27. Wu $\mathrm{H}$, et al. Advantages of single-nucleus over single-cell RNA sequencing of adult kidney: rare cell types and novel cell states revealed in fibrosis. J Am Soc Nephrol. 2019;30(1):23-32.

28. Shved N, et al. Transcriptome-based network analysis reveals renal cell type-specific dysregulation of hypoxia-associated transcripts. Sci Rep. 2017;7(1):8576.

29. Klinkhammer BM, et al. Cellular and molecular mechanisms of kidney injury in 2,8-dihydroxyadenine nephropathy. J Am Soc Nephrol. 2020;31(4):799-816.

30. Sun $\mathrm{Q}$, et al. Elastin imaging enables noninvasive staging and treatment monitoring of kidney fibrosis. Sci Transl Med. 2019;11(486):eaat4865.

31. Cosgrove D, et al. Collagen COL4A3 knockout: a mouse model for autosomal Alport syndrome. Genes Dev. 1996;10(23):2981-2992.

32. Ding $\mathrm{H}$, et al. Activin A induces skeletal muscle catabolism via p $38 \beta$ mitogen-activated protein kinase. JCachexia Sarcopenia Muscle. 2017;8(2):202-212.

33. Qin $\mathrm{W}$, et al. Identification of functional glucocorticoid response elements in the mouse FoxO1 promoter. Biochem Biophys Res Commun. 2014;450(2):979-983.

34. Sandri M, et al. Foxo transcription factors induce the atrophy-related ubiquitin ligase atrogin-1 and cause skeletal muscle atrophy. Cell. 2004;117(3):399-412.

35. McPherron AC, et al. Regulation of skeletal muscle mass in mice by a new TGF-beta superfamily member. Nature. 1997;387(6628):83-90.

36. Souza TA, et al. Proteomic identification and functional validation of activins and bone morphogenetic protein 11 as candidate novel muscle mass regulators. Mol Endocrinol. 2008;22(12):2689-2702.

37. Droujinine IA, Perrimon N. Defining the interorgan communication network: systemic coordination of organismal cellular processes under homeostasis and localized stress. Front Cell Infect Microbiol. 2013;3:82.

38. Tezze C, et al. Age-associated loss of OPA1 in muscle impacts muscle mass, metabolic homeostasis, systemic inflammation, and epithelial senescence. Cell Metab. 2017;25(6):1374-1389.

39. Geminard C, et al. Remote control of insulin secretion by fat cells in Drosophila. Cell Metab. 2009;10(3):199-207.

40. Febbraio MA, et al. Interleukin-6 is a novel factor mediating glucose homeostasis during skeletal muscle contraction. Diabetes. 2004;53(7):1643-1648.

41. Bostrom P, et al. A PGC1- $\alpha$-dependent myokine that drives brown-fat-like development of white fat and thermogenesis. Nature. 2012;481(7382):463-468.

42. Ahima RS, et al. Role of leptin in the neuroendocrine response to fasting. Nature. 1996;382(6588):250-252.

43. Garibotto $\mathrm{G}$, et al. Insulin sensitivity of muscle protein metabolism is altered in patients with chronic kidney disease and metabolic acidosis.
Kidney Int. 2015;88(6):1419-1426.

44. Barraclough KA, et al. Coronary artery calcification scores in patients with chronic kidney disease prior to dialysis: reliability as a trial outcome measure. Nephrol Dial Transplant. 2008;23(10):3199-3205.

45. Sette LH, Almeida Lopes EP. Liver enzymes serum levels in patients with chronic kidney disease on hemodialysis: a comprehensive review. Clinics (Sao Paulo). 2014;69(4):271-278.

46. Dong J, et al. The pathway to muscle fibrosis depends on myostatin stimulating the differentiation of fibro/adipogenic progenitor cells in chronic kidney disease. Kidney Int. 2017;91(1):119-128.

47. Abramowitz MK, et al. Skeletal muscle fibrosis is associated with decreased muscle inflammation and weakness in patients with chronic kidney disease. Am J Physiol Renal Physiol. 2018;315(6):F1658-F1669.

48. Alyodawi K, et al. Compression of morbidity in a progeroid mouse model through the attenuation of myostatin/activin signalling. J Cachexia Sarcopenia Muscle. 2019;10(3):662-686.

49. Tampe D, Zeisberg M. Potential approaches to reverse or repair renal fibrosis. Nat Rev Nephrol. 2014;10(4):226-237.

50 . Grgic I, et al. The origin of interstitial myofibroblasts in chronic kidney disease. Pediatr Nephrol. 2012;27(2):183-193.

51. Wynn TA. Cellular and molecular mechanisms of fibrosis. J Pathol. 2008;214(2):199-210.

52. Lin SL, et al. Pericytes and perivascular fibroblasts are the primary source of collagen-producing cells in obstructive fibrosis of the kidney. Am J Pathol. 2008;173(6):1617-1627.

53. Humphreys BD, et al. Fate tracing reveals the pericyte and not epithelial origin of myofibroblasts in kidney fibrosis. Am J Pathol. 2010;176(1):85-97.

54. Chen YT, et al. Platelet-derived growth factor receptor signaling activates pericyte-myofibroblast transition in obstructive and post-ischemic kidney fibrosis. Kidney Int 2011;80(11):1170-1181.

55. Chang FC, et al. Novel insights into pericytemyofibroblast transition and therapeutic targets in renal fibrosis. J Formos Med Assoc. 2012;111(11):589-598.

56. Asada N, et al. Dysfunction of fibroblasts of extrarenal origin underlies renal fibrosis and renal anemia in mice. JClin Invest. 2011;121(10):3981-3990.

57. Grgic I, et al. Translational profiles of medullary myofibroblasts during kidney fibrosis. J Am Soc Nephrol. 2014;25(9):1979-1990.

58. Xia Y, Schneyer AL. The biology of activin: recen advances in structure, regulation and function. JEndocrinol. 2009;202(1):1-12.

59. Tsuchida K, et al. Activin signaling as an emerging target for therapeutic interventions. Cell Commun Signal. 2009;7:15.

60. Latres E, et al. Activin A more prominently regulates muscle mass in primates than does GDF8. Nat Commun. 2017;8:15153.

61. Zhou X, et al. Reversal of cancer cachexia and muscle wasting by ActRIIB antagonism leads to prolonged survival. Cell. 2010;142(4):531-543.
62. Lach-Trifilieff E, et al. An antibody blocking activin type II receptors induces strong skeletal muscle hypertrophy and protects from atrophy. Mol Cell Biol. 2014;34(4):606-618.

63. Lee SJ, et al. Targeting myostatin/activin A protects against skeletal muscle and bone loss during spaceflight. Proc Natl Acad Sci U S A. 2020;117(38):23942-23951.

64. Rooks D, et al. Treatment of sarcopenia with bimagrumab: results from a phase II, randomized, controlled, proof-of-concept study. J Am Geriatr Soc. 2017;65(9):1988-1995.

65. Hatakeyama S, et al. ActRII blockade protects mice from cancer cachexia and prolongs survival in the presence of anti-cancer treatments. Skelet Muscle. 2016;6:26.

66. Sherman ML, et al. Multiple-dose, safety, pharmacokinetic, and pharmacodynamic study of sotatercept (ActRIIA-IgG1), a novel erythropoietic agent, in healthy postmenopausal women. JClin Pharmacol. 2013;53(11):1121-1130.

67. Amato AA, et al. Treatment of sporadic inclusion body myositis with bimagrumab. Neurology. 2014;83(24):2239-2246.

68. Koncarevic A, et al. A novel therapeutic approach to treating obesity through modulation of TGF $\beta$ signaling. Endocrinology. 2012;153(7):3133-3146

69. Lee SJ, et al. Functional redundancy of type I and type II receptors in the regulation of skeletal muscle growth by myostatin and activin A. Proc Natl Acad Sci U S A. 2020;117(49):30907-30917.

70. Peng $\mathrm{H}$, et al. Myokine mediated muscle-kidney crosstalk suppresses metabolic reprogramming and fibrosis in damaged kidneys. Nat Commun. 2017;8(1):1493.

71. Braun TP, et al. Central nervous system inflammation induces muscle atrophy via activation of the hypothalamic-pituitary-adrenal axis. JExp Med. 2011;208(12):2449-2463.

72. Enoki $Y$, et al. Potential therapeutic interventions for chronic kidney disease-associated sarcopenia via indoxyl sulfate-induced mitochondrial dysfunction. JCachexia Sarcopenia Muscle. 2017;8(5):735-747.

73. Watanabe H, et al. Sarcopenia in chronic kidney disease: factors, mechanisms, and therapeutic interventions. Biol Pharm Bull. 2019;42(9):1437-1445.

74. Gregorevic P, et al. Systemic delivery of genes to striated muscles using adeno-associated viral vectors. Nat Med. 2004;10(8):828-834.

75. Aurnhammer C, et al. Universal real-time PCR for the detection and quantification of adenoassociated virus serotype 2-derived inverted terminal repeat sequences. Hum Gene Ther Methods. 2012;23(1):18-28.

76. Piccolo SR, et al. A single-sample microarray normalization method to facilitate personalized-medicine workflows. Genomics. 2012;100(6):337-344.

77. Menon R, et al. Single-cell analysis of progenitor cell dynamics and lineage specification in the human fetal kidney. Development. 2018;145(16): dev164038.

78. Anders S, et al. HTSeq-a Python framework to work with high-throughput sequencing data. Bioinformatics. 2015;31(2):166-169.

79. Menon R, et al. Single cell transcriptomics identifies focal segmental glomerulosclerosis 
remission endothelial biomarker. JCI Insight. 2020;5(6):e133267.

80. Cohen CD, et al. Quantitative gene expression analysis in renal biopsies: a novel protocol for a high-throughput multicenter application. Kidney Int. 2002;61(1):133-140.

81. Tusher VG, et al. Significance analysis of microarrays applied to the ionizing radiation response.
Proc Natl Acad Sci U S A. 2001;98(9):5116-5121. 82. Levey AS, et al. A new equation to estimate glomerular filtration rate. Ann Intern Med. 2009;150(9):604-612.

83. Stuart T, et al. Comprehensive integration of single-cell data. Cell. 2019;177(7):1888-1902.

84. Park J, et al. Single-cell transcriptomics of the mouse kidney reveals potential cellular targets of kidney disease. Science. 2018;360(6390):758-763.

85. Wang F, et al. RNAscope: a novel in situ RNA analysis platform for formalin-fixed, paraffin-embedded tissues. J Mol Diagn. 2012;14(1):22-29.

86. Mammucari C, et al. FoxO3 controls autophagy in skeletal muscle in vivo. Cell Metab. 2007;6(6):458-471. 